\title{
Integrated single-cell analysis unveils diverging immune features of COVID-19, influenza and other community-acquired pneumonia.
}

\author{
Alex Schuurman ( $\nabla$ a.r.schuurman@amsterdamumc.nl ) \\ Amsterdam UMC - location AMC https://orcid.org/0000-0001-9322-1117 \\ Tom Reijnders \\ Amsterdam UMC - location AMC https://orcid.org/0000-0002-1764-0114 \\ Anno Saris \\ Amsterdam UMC
}

Ivan Ramirez-Moral

Amsterdam UMC - location AMC

Michiel Schinkel

Amsterdam UMC - location AMC

Justin de Brabander

Amsterdam UMC - location AMC

Christine van Linge

Amsterdam UMC - location AMC

Louis Vermeulen

Amsterdam UMC https://orcid.org/0000-0002-6066-789X

\section{Brendon Scicluna}

Amsterdam UMC - location AMC

\section{Willem Wiersinga}

Department of Internal Medicine, Division of Infectious Diseases, Amsterdam University Medical Centers-Location AMC, University of Amsterdam, Amsterdam, the Netherlands

\section{Felipe A. Vieira Braga}

Amsterdam Medical Centre https://orcid.org/0000-0003-0206-9258

Tom van der Poll

Amsterdam UMC https://orcid.org/0000-0002-9199-5079

\section{Article}

Keywords: community-acquired pneumonia (CAP), COVID-19, influenza, SARS-CoV-2

Posted Date: February 24th, 2021 
DOI: https://doi.org/10.21203/rs.3.rs-226162/v1

License: (c) (1) This work is licensed under a Creative Commons Attribution 4.0 International License. Read Full License 
Integrated single-cell analysis unveils diverging immune features of COVID-19, influenza and other community-acquired pneumonia.

Alex R. Schuurman ${ }^{1,2^{*}}$, Tom D. Y. Reijnders ${ }^{1,2^{*}}$, Anno Saris ${ }^{1,2}$, Ivan Ramirez Moral ${ }^{1,2}$, Michiel Schinkel $^{1,2}$, Justin de Brabander ${ }^{1,2}$, Christine van Linge ${ }^{1,2}$, Louis Vermeulen ${ }^{3}$, Brendon P. Scicluna $^{1,4}$, W. Joost Wiersinga ${ }^{1,2,5}$, Felipe A. Vieira Braga ${ }^{3,6}$, Tom van der Poll ${ }^{1,2,5,6,7}$

\section{Affiliations:}

1 Center for Experimental and Molecular Medicine, Amsterdam UMC, Academic Medical Center, University of Amsterdam, Amsterdam, Netherlands.

2 Amsterdam Institute for Infection and Immunity, Amsterdam UMC, Amsterdam, The Netherlands.

3 Laboratory for Experimental Oncology and Radiobiology, Center for Experimental and Molecular Medicine, Cancer Center Amsterdam and Amsterdam Gastroenterology and Metabolism, Amsterdam UMC, Academic Medical Center, University of Amsterdam, Amsterdam, The Netherlands.

4 Department of Clinical Epidemiology, Biostatistics and Bioinformatics, Amsterdam UMC, Academic Medical Center, University of Amsterdam, Amsterdam, The Netherlands.

5 Division of Infectious Diseases, Amsterdam UMC, Academic Medical Center, University of Amsterdam, Amsterdam, The Netherlands.

6 Senior author

7 Corresponding author: t.vanderpoll@amsterdamumc.nl

* These authors contributed equally 


\begin{abstract}
The exact immunopathophysiology of community-acquired pneumonia (CAP) caused by SARS-CoV-2 (COVID-19) remains clouded by methodological heterogeneity and a lack of relevant disease controls. The absence of single-cell investigations in the broader population of patients with CAP renders it difficult to distinguish immune features unique to COVID-19 from the common characteristics of a dysregulated host response to pneumonia. We performed integrated single-cell transcriptomic and proteomic analyses in PBMCs from a matched cohort of eight patients with COVID-19, eight patients with CAP caused by Influenza A or other pathogens, and four non-infectious control subjects. Using this balanced, multi-omics approach we describe shared and diverging transcriptional and phenotypic patterns - including increased levels of type I interferon stimulated NK cells in COVID-19, cytotoxic CD8 T EMRA cells in both COVID-19 and influenza, and distinctive monocyte compositions between all groups and thereby expand our understanding of the peripheral immune response in different etiologies of pneumonia.
\end{abstract}




\section{Introduction}

The devastating impact of COVID-19 on both a global and interpersonal level is unprecedented in living memory. Patients with severe COVID-19 - caused by the SARS-CoV-2 virus develop bilateral pneumonia, profound systemic inflammation, and organ failure reminiscent of sepsis ${ }^{1-3}$. Within one year of its discovery, the world has seen hundreds of thousands excess deaths ${ }^{4}$.

Progression to life-threatening disease is mediated by a dysfunctional and excessive host immune response to SARS-CoV-2 $2^{5}$. Distinct patterns are beginning to emerge in the characteristics of this immune response in hospitalized patients: lymphopenia ${ }^{1,6}$, a role for activated and exhausted $\mathrm{T}$ cells $\mathrm{s}^{7,8}$, hyperinflammatory monocytes with reduced antigen presenting capacity ${ }^{9,10}$, delayed or dysfunctional interferon responses ${ }^{11-13}$, and expansion of plasmablasts and suppressive immature neutrophils in severe diseas ${ }^{9,14}$.

Thus far, most reports on deep immunophenotyping of patients with COVID-19 have been variable (both within and between studies) in important determinants of the immune response, such as disease severity, time of sampling, and age and sex of the participants. Key interventions in intensive care units, such as mechanical ventilation and vasopressors, exacerbate this problem through rapid and profound immunomodulatory effects ${ }^{15}$. These factors make it difficult to separate the true signal of COVID-19 immunopathophysiology from the noise of methodological heterogeneity and the potential distortions of confounding and bias.

Crucially, despite early observations that many of the immune disturbances, clinical symptoms and organ dysfunctions in COVID-19 can also occur in other infections, most studies lack disease controls ${ }^{3,16}$. The direct comparison of COVID-19 with another, well matched infectious disease state is urgently needed to distinguish truly specific immune features from the common characteristics of a dysregulated host response to infection. However, while several studies 
reported on the immunological response during COVID-19 pneumonia at the single cell level, such investigations are currently lacking for the broader population of community-acquired pneumonia $(\mathrm{CAP})^{9,11-13,17,18}$.

Here, we performed CITE-Seq (Cellular Indexing of Transcriptomes and Epitopes by Sequencing ${ }^{19}$ ) on peripheral blood mononuclear cells (PBMCs) from age-, sex- and disease severity-matched patients with CAP caused by either SARS-CoV-2, Influenza A, or other pathogens admitted to a non-intensive care ward. By integrating single cell RNA-sequencing with highly multiplexed surface protein marker detection - akin to classical flow cytometry we create a high-resolution snapshot of cellular phenotypes and functional states, offering insight into the peripheral immune features of different etiologies of pneumonia. 


\section{Results}

\section{Patient characteristics and clinical outcomes}

We profiled PBMCs from eight patients hospitalized for CAP caused by SARS-CoV-2 (henceforth referred to as COVID-19), eight patients hospitalized for CAP caused by either Influenza A or other pathogens (all sampled before the start of the COVID-19 pandemic) and four non-infectious control subjects visiting the outpatient clinic without signs of acute infection (Table 1 for summary data, Supplementary Table 1 for individual patient data). We matched the groups for age, sex, and - the patients - for disease severity using the Modified Early Warning Score ${ }^{20,21}$. Samples from patients were obtained within 48 hours of admission to the general hospital ward, none of the patients received systemic corticosteroid therapy prior to sampling. Patients with COVID-19 reported a longer duration of symptoms prior to hospital admission. Lymphocyte counts were reduced in both patient groups but not significantly different, whereas neutrophil counts were significantly higher in patients with non-COVID-19 CAP. All patients classified as COVID-19 had a positive PCR for SARS-CoV-2. In the patients without COVID-19, three had a positive PCR for Influenza A - one of whom also had a positive blood culture for Streptococcus pneumoniae - and one patient had a positive sputum culture for Haemophilus influenzae. In subsequent analyses, we refer to the three patients infected with Influenza A as CAP-flu, and the remaining five patients as CAP-other. None of the patients were admitted to the intensive care unit during their hospital stay, one patient with COVID-19 died in the hospital as a result of the disease.

Highly cytotoxic CD8 EMRA-like T cells and type I interferon-stimulated NK cells characterize COVID-19 patients

An overview of the experimental setup is depicted in Fig. 1a. Post quality control we analyzed 16,192 cells from all profiled samples. Throughout this study, we infer disease-specific effects 
in part by examining the transcriptional states of cell clusters that are proportionally expanded within a disease group. We evaluated proportional differences in cell clusters between groups with the Pearson's residual of the chi-squared test, in which comparisons with more than two residuals of difference were considered biologically relevant. All gene expression analyses were corrected for multiple testing using the Benjamini-Hochberg $(\mathrm{BH})$ method, with significance defined throughout as an adjusted $\mathrm{p}<0.05$.

We first explored the peripheral blood immune response of patients with COVID-19 pneumonia and non-infectious controls. Uniform Manifold Approximation and Projection (UMAP) dimensionality reduction of the transcriptome of all individual cells from these subjects identified thirteen clusters of myeloid and lymphoid immune cells (Figs. 1b,c). The top differentially expressed genes (DEGs) between these clusters can be found in Supplementary Fig. 1a. Cell surface protein expression levels of classical lineage-defining markers (e.g. CD3, CD4, CD8, CD14, CD19) were consistent with the transcriptome-based clusters (Supplementary Figs. 1b and c).

When compared with age- and sex-matched non-infectious control subjects, patients with COVID-19 exhibited stark differences in the proportional composition of cell clusters, including a decrease in monocytes and memory B cells, and an increase in naive B cells (Figs. 1c,d). While some lymphocyte clusters were relatively decreased, we found a striking proportional increase in a specific T cell cluster and NK cells in patients with COVID-19. This expanded T cell cluster mainly expressed low levels of surface CD27 and variable levels of CD45RA (Supplementary Fig. 1d), resembling the terminally differentiated effector memory re-expressing CD45RA (EMRA) phenotype as defined by classical flow cytometry (CCR7$\mathrm{CD} 27-\mathrm{CD} 45 \mathrm{RA}+{ }^{22}$ ). In fact, these cells (that we will henceforth refer to as CD8 EMRA-like T 
cells) formed a cluster almost entirely composed of cells from patients with COVID-19 (Fig. 1c and Supplementary Fig. 1e). As the CD8 EMRA-like T cell and NK cell clusters showed the most prominent proportional increase in COVID-19 when compared with non-infectious controls (Fig. 1D), we next sought to uncover the identity and transcriptional state of these clusters.

In all $\mathrm{T}$ and $\mathrm{NK}$ cell clusters, we examined the expression of canonical genes and the top DEGs derived from comparing the CD8 EMRA-like and CD8 EM (effector memory) clusters (Fig. 1e for selected genes, Supplementary Fig. 2a for the full list of DEGs). At the transcript level, the EMRA-like T cells expressed low levels of the canonical markers $I L 7 R$ (encoding for CD127) and CCR7. The CD8 EMRA-like T cells exhibited remarkably high expression of genes related to cytotoxicity and killing - seemingly on par with NK cells - such as GZMB, GZMH, PRF1, and $N K G 7$. These cells were also marked by the high expression of ITGB1 (CD29), which has recently been described as a marker of $\mathrm{T}$ cells with increased cytotoxic potential ${ }^{23}$.

We next examined the top DEGs between the CD8 EMRA-like T cell and NK cell clusters. The NK cell cluster was enriched in genes corresponding to type I and, to a lesser extent, type II interferon signaling pathways (Supplementary Figs. 2b,c). We then compared the expression levels of genes within the NK cell cluster between patients with COVID-19 and control subjects (Fig. 1f) and discovered marked upregulation of pathways related to a type I interferon response and other antiviral responses in patients with COVID-19 (Biological Process Gene Ontology pathway analysis; Fig. 1g). Transposing this NK cell cluster-derived type I interferon response signature to the other cell clusters revealed a broad pattern of type I interferon responses across all clusters, statistically significant in several clusters, including NK cells (two-sided Wilcoxon rank-sum test $p<0.05$; Fig. 1h). Taken together, when compared with matched non-infectious 
controls, patients with COVID-19 exhibited a marked proportional increase in CD8 EMRAlike T cells and type I interferon-stimulated NK cells, both with high cytotoxic potential.

Differential composition of lymphoid cells in patients with CAP-flu and patients with CAPother

We next investigated the immune features of patients with CAP caused by Influenza A (CAPflu) or other pathogens (CAP-other), as compared with matched non-infectious controls. UMAP dimensionality reduction revealed thirteen clusters of cells (Figs. 2a,c; and Supplementary Figs 3a-c for top DEGs and surface protein expression). Akin to what we observed in patients with COVID-19, patients with CAP-flu showed a proportional increase of CD8 EMRA-like T cell and NK cell clusters (Figs. 2b,c; cluster distribution per subject in Supplementary Fig. 3d). Patients with CAP-other had higher levels of the CD8 EM and naïve T cell clusters (Fig. 2c). Both CAP-flu and CAP-other patients showed a decrease in memory B cells when compared with control subjects (Fig. 2c).

To elucidate the transcriptional states of the differentially expanded $\mathrm{T}$ cell subsets between CAP-flu and CAP-other patients, we examined the top DEGs between CD8 EM T and CD8 EMRA-like cells (Fig. 2d): among the top upregulated genes in the CD8 EM-cluster we identified GZMK and genes related to MHC class II (HLD-DRB1 and CD74). CD8 EMRA-like T cells were characterized by genes related to cytotoxicity and activation signals, such as GNLY, GZMB, TYROBP, and FGFBP, which were also highly expressed by NK cells. We observed few specific gene differences in EMRA-like T cells and NK cells from patients with CAP-flu and CAP-other when compared with controls (Supplementary Figs. 4a-d). Thus, patients with CAP-flu harbored higher proportions of activated and cytotoxic CD8 EMRA-like T cells and 
NK cells, while the lymphocyte composition in patients with CAP was characterized by higher proportions of the CD8 EM and naïve T cell clusters.

Classical monocytes from patients with CAP-flu show concurrent signs of inflammation and immune suppression

We next focused on monocytes, because specific clusters were positively associated with either CAP-flu or CAP-other. Patients with CAP-flu exhibited a clear increase in classical monocytes when compared with patients with CAP-other and control subjects (Figs. 2b,c). The proportional increase in the intermediate monocyte cluster in CAP-flu is unlikely to represent a disease-specific process, as these cells were almost entirely derived from one patient (Supplementary Fig. 3d). Patients with CAP-other demonstrated a clear increase in nonclassical monocytes, contributing nearly all cells in this cluster (Fig. 2b and Supplementary Fig. 3d). Low absolute dendritic cell (DC) counts precluded a valid comparison between disease states.

To further explore the myeloid cell clusters that were differentially expanded between CAP-flu and CAP-other, we assessed the surface marker expression of CD14 and CD16 (Supplementary Fig. 4e). In line with their transcriptome, classical monocytes were mostly CD14+ and CD16-, whereas non-classical monocytes were $\mathrm{CD} 14^{\mathrm{dim}}$ and CD16+. Intermediate monocytes and DCs showed variable CD14 expression, and mostly low CD16 expression (Supplementary Fig. 4e). Analysis of protein expression identified differential expression patterns of CD11b, CD11c and CD33, which further consolidated the identity of these clusters (Fig. 2e) ${ }^{24}$. Differential gene expression analysis directly comparing classical with non-classical monocytes and Gene Ontology pathways corresponding to the upregulated genes are depicted in Supplementary Figs. 4f-h. 
As cell numbers within the classical monocyte cluster were higher among patients with CAPflu (Fig. 2c), we examined DEGs within this cluster between CAP-flu and control subjects (Fig. 2f). Classical monocytes from patients with CAP-flu displayed upregulation of a variety of genes involved in proinflammatory processes, such as $E G R-1^{25}, F K B 5^{26}$, and $A R E G^{27}$. Classical monocytes also transcribed several genes encoding for the S100 protein family, such as S100A4, S100A8, S100A9 and S100A12. Strikingly, we observed a concurrent substantially reduced expression of genes related to MHC class II (Fig. 2f), a quintessential feature of sepsisinduced immune suppression that has been associated with secondary infections and long-term mortality ${ }^{28,29}$. Gene ontology enrichment of these downregulated genes confirmed downregulation of pathways related to antigen presentation (Fig. $2 \mathrm{~g}$ ) and this pattern was also visible - albeit to a lesser extent and not statistically significant - in non-classical monocytes from patients with CAP-flu and classical monocytes from patients with CAP-other (two-sided Kruskal-Wallis test with post-hoc Dunn's test BH-adjusted $\mathrm{p}<0.05$ for CAP-flu versus control in classical monocytes; Fig. 2h). Reduced HLA-DR expression on the cell surface of classical monocytes in patients with CAP-flu confirmed this transcriptional pattern at the protein level (Fig. 2i). Taken together, we report clearly diverging monocyte compositions in patients with CAP-flu and CAP-other, with classical monocytes in CAP-flu expressing pro-inflammatory genes while simultaneously showing immune suppressive features.

Divergent composition of major immune cell types in patients with COVID-19, CAP-flu and CAP-other

Next, we directly compared COVID-19, CAP-flu and CAP-other to delineate the shared and unique immune features between these disease groups. UMAP dimensionality reduction of all cells of these groups revealed fifteen clusters of myeloid and lymphoid cells (the full UMAP of 
all identified clusters, the distribution per individual patient, and the differentially expressed genes and proteins between all clusters are depicted in Supplementary Figs. 5 and 6). To create an overview of the differences in immune composition between disease states, we first grouped these clusters into metaclusters representing the major cell types: T cells, NK cells, B cells, monocytes, and platelets (Figs. 3a-c; canonical genes and lineage-defining surface markers in Figs. $3 \mathrm{~d}$ and $3 \mathrm{e}$, respectively). Comparing the proportional composition of immune cells between diseases revealed a significant expansion of NK cells in COVID-19, an expansion of monocytes in CAP-flu, and an overall expansion of T cells in CAP-other (Fig. 3c). Proportions of B cells were comparable between the groups. As we previously noted reduced HLA-DR surface protein expression in classical monocytes of patients with CAP-flu, we compared expression of this protein in all monocytes between the three disease states (Fig. 3f). Overall monocyte HLA-DR surface protein expression in COVID-19 was on par with CAP-flu, and lower than in CAP-other.

Differences in transcriptional signature between COVID-19 and CAP-flu in T and NK cells

To ascertain differences in the T and NK cell compositions between COVID-19, CAP-flu and CAP-other, we further examined clusters within these metaclusters (Fig. 4a; see Supplementary Fig. 5a for full UMAP of all identified clusters, and Supplementary Fig. 5b for clusters per individual patient). We observed fewer cells in the naive CD4/CD8 T cell cluster in COVID19 and CAP-flu as compared with CAP-other, whereas the CD8 EMRA-like 2 T cell cluster was clearly expanded in CAP-flu (Fig. 4b; see Supplementary Fig. 5c for the correlation plot of all identified clusters). Patients with CAP-other exhibited a proportional increase of CD8 EM T cells and had the most naive T cells.

To infer functional differences in T cell subsets, we looked at the top DEGs between the two 
EMRA-like T cell clusters (Fig. 4c). We noted increased expression of genes related to cytotoxicity, activation and inflammation - such as GZMB, GNLY, TYROBP, HOPX, and CCL5 - in the CD8 EMRA-like 2 cluster that was expanded in CAP-flu. The contrast in expression levels of these genes was especially apparent in comparison with the CD8 EM T cell cluster predominant in CAP-other, indicating that patients with a known viral infection harbor more activated CD8 T cells.

In line with high GNLY expression in the EMRA-like $2 \mathrm{~T}$ cell cluster expanded in CAP-flu, we found a higher proportion of granulysin+ NK cells in CAP-flu, whereas the other (granulysin negative) NK cell cluster was highest in COVID-19 (Figs. 4b,c). Interestingly, the NK cell cluster expanded in COVID-19 expressed higher levels of the interferon-stimulated gene IFITM3 (Fig. 4d) than the cluster expanded in CAP-flu. This high expression was even more apparent when comparing cells within the granulysin+ NK cell cluster between patients with COVID-19 and CAP-flu (Fig. 4d). Gene ontology enrichment of the genes upregulated in granulysin+ NK cells from patients with COVID-19 exposed a clear type I interferon response (Fig. 4f). When comparing this pathway between disease groups in all T and NK cell clusters, we found a this pronounced type I interferon signature among virtually all clusters in patients with COVID-19, particularly in the EMRA-like T cell clusters and both NK cell clusters (Figs. 4g,h, statistically significant in the CD8 EMRA-like 2 and+ granulysin+ NK cell clusters, twosided Kruskal-Wallis test with post-hoc Dunn's test BH-adjusted $\mathrm{p}<0.05$ ). To summarize, while both groups of patients with known viral infections exhibited high proportions of circulating NK cells and activated (EMRA-like) CD8 T cells when compared with CAP-other, COVID-19 was distinguished from other etiologies of CAP by a pronounced type I interferon signaling transcriptional signature. 
Next, we focused on the differences in monocyte clusters between COVID-19, CAP-flu and CAP-other (Fig. 5a; Supplementary Figs. 5a-c). Classical monocytes were proportionally expanded in both CAP groups, albeit primarily in patients with CAP-flu (Fig. 5b). The intermediate monocyte cluster was prominently expanded in patients with COVID-19, whereas the non-classical monocyte cluster showed a clear expansion in the CAP-other patients.

To explore the transcriptional states of the diverging monocyte compositions in disease states, we looked at the top DEGs between the monocyte clusters, and the pathways related to the upregulated genes within each cluster (Figs. 5c-f). In classical monocytes (proportionally higher in patients with CAP-flu) the majority of upregulated genes was related to ribosomes and viral transcription (Figs. 5c,d). The intermediate monocytes (consistently present in COVID-19) were characterized by a pronounced antiviral, interferon-driven response, as illustrated by the upregulation of both interferon inducible- and stimulated genes (IFI6, IFI27, IFI44L, and ISG15, SIGLEC1; Figs. 5c,e). The high expression of APOBEC $3 A$ and $M X 1$, involved in restricting viral activity in monocytes through RNA editing ${ }^{30,31}$, underlined this increased antiviral potential. The upregulated DEGs of the non-classical monocyte cluster (predominantly present in CAP-other) were more heterogeneous: they expressed genes involved in MHC class II antigen-presenting, Fc receptors $(F C G R 3 A, F C E R 1 G)$, the complement system $(C 1 Q A$, CFD), and multiple genes associated with cell activation (AIF1, LST1, SMIM25; Figs. 5c,f). 


\section{Discussion}

We performed single-cell transcriptomic and proteomic analyses in PBMCs from a matched cohort of eight patients with CAP caused by SARS-CoV-2 (COVID-19), eight patients with CAP caused by Influenza A or other pathogens, and four non-infectious control subjects, all without overt prior immune disturbances from comorbidities or medication. Using this approach we provide a high-resolution overview of the peripheral immune features of COVID19 and contrast this with the broader population of patients with CAP. Our investigation on the diverging peripheral immune features of CAP due to different infectious etiologies provides a framework for interpreting the role of $\mathrm{T}$ cells, NK cells and monocytes in the immunopathophysiology of COVID-19.

Deciphering the immune response of COVID-19 from the vast amounts of data generated thus far poses a unique challenge. Whereas our knowledge of the pathophysiological basis of other diseases is often derived from slowly-accumulated data generated in controlled settings, including in vitro and animal models, the scientific community now has to distill the true signal of disease immunopathophysiology largely from rapidly expanding and highly diverse clinical studies utilizing a variety of methods. A multi-dimensional snapshot obtained via -omics technologies is only useful if its target - and thus to whom the immunological findings may generalize - is clearly defined. We aimed to capture an undistorted view of the immune disturbances in hospitalized patients with COVID-19 (and other etiologies of CAP) by only including patients in a non-intensive care ward within 48 hours of hospital admission. With a matched and restricted design, we attempted to limit confounding by important determinants of the immune response, such as disease severity, age and sex, and invasive therapeutic interventions. 
Despite overall lymphopenia in our population of patients with COVID-19, consistently described in earlier studies ${ }^{5}$, we report a proportional increase in cells that resemble CD8 EMRA T cells (referred to as the CD8 EMRA-like T cell clusters) with a pronounced cytotoxic transcriptional signature. Importantly, CAP-flu - but not CAP-other - was associated with a similar CD8 EMRA T cell response, suggesting that this is induced by viral infection in general rather than by SARS-CoV-2 specifically. CD8 EMRA T cells effectively kill virus-infected cells, have a varying proliferation capacity, and (together with CD8 EM T cells) represent the predominant phenotype in circulating CD8 $\mathrm{T}$ cells specific for respiratory viruses in adults $^{22,32,33}$. Other studies in COVID-19, either overall or on antigen-specific CD8 T cells, similarly reported on CD8 T cells that were highly activated ${ }^{8,11,34,35}$, had increased cytotoxic potential $^{11,35}$, and exhibited characteristics of EMRA (or sometimes EM) cells s,34,36,37 $^{8}$ These T cells may be clonally expanded in moderate, but not severe, disease ${ }^{11,38}$. Taken together, these findings may point towards an important role for cytotoxic T cells with EMRA-like features in controlling the virus both in influenza and COVID-19 pneumonia, although it would be informative to contrast these findings with the $\mathrm{T}$ cell response in infections that do not require hospitalization.

Whereas T cell lymphopenia has been a ubiquitous finding, the effect of COVID-19 on NK cell numbers is more ambiguous, with cytopenia often limited to the most severe cases ${ }^{11,13,35,39}$. NK cells are innate lymphoid cells generally critical in the early stages of controlling viral infections, but may also contribute to immunopathology ${ }^{40}$. We report a proportional increase in highly cytotoxic NK cells with a type I interferon transcriptional signature in COVID-19. Patients with CAP-flu - but not with CAP-other - also displayed an expansion of NK cells when compared with non-infectious control subjects, but this was less pronounced when compared directly with COVID-19. While the cytotoxic and interferon-responsive 
transcriptional features are in line with earlier findings, an expansion of NK cells has not been described in previous COVID-19 single-cell reports ${ }^{11,13,18}$. However, Wilk et al. reported that CD56 ${ }^{\mathrm{dim}} \mathrm{NK}$ cells - key in the cytotoxic antiviral response - were maintained in moderate disease, whereas the CD56 $6^{\text {bright }} \mathrm{NK}$ cells (prone to produce pro-inflammatory cytokines such as IFN- $\gamma$ ) were depleted in both moderate and severe disease ${ }^{13}$. COVID-19 has been hypothesized to either induce hyperresponsive NK cells that exacerbate lung-destructive inflammation through production of IFN- $\gamma$; or to hamper effective viral clearance by NK cells, such as through suppressing type I interferon responses and reducing circulating cell numbers ${ }^{40}$. In influenza, depletion of NK cells has been implicated only in severe disease, with normal or even increased NK cell counts after mild disease or vaccination ${ }^{41,42}$. While our results partly deviate from previous reports, they could indicate a relatively adequate NK cell response in this population of non-intensive care unit patients with COVID-19 and CAP-flu.

Monocytes are key players in the innate immune response to pathogens, and have been reported to respond divergently to different types of RNA viruses ${ }^{43}$. We found striking proportional and transcriptional differences in monocyte subsets between the disease groups: in COVID-19, intermediate monocytes were expanded with a clear antiviral and type I interferon signature, while in CAP-flu, classical monocytes predominated and were characterized by ribosomal translational activity. An enhanced type I interferon response in monocytes of patients with COVID-19 has previously been reported ${ }^{9,11}$, but interestingly CAP-flu did not provoke such a strong antiviral response in the expanded monocyte subset. Interferon evasion and hijacking of the host's ribosomal translational machinery are hallmarks of active viral replication ${ }^{44-46}$. While both Influenza and SARS-CoV-2 can infect human monocytes ${ }^{47,48}$, the clear transcriptomic difference in our population could indicate that monocytes in patients with CAP-flu - but not COVID-19 - harbored intracellular viruses. In CAP-other, non-classical monocytes 
preponderated, characterized by enhanced expression of genes involved in MHC-II signaling, which - together with the differential lymphocyte responses discussed above - clearly suggests that the peripheral immune response in CAP depends at least in part on the type of causative pathogen.

Our findings, pointing to a pronounced transcriptional type I interferon response of T cells, NK cells, and monocytes in COVID-19 versus Influenza A are contrasted by Lee et al., who reported a more pronounced type I interferon response in influenza ${ }^{12}$. This discrepancy may result from differences in diseases severity: all influenza cases in their cohort were classified as 'severe', whereas the COVID-19 cases included mild and asymptomatic cases ${ }^{12}$. Zhu et al. also found a clear type I interferon response signature in T, B and NK cells in longitudinal samples of patients with COVID-19 (four mild, one severe) when compared with controls ${ }^{18}$. Increased expression of IFNARI and IRF3 in patients with COVID-19 when compared with two patients infected with Influenza A also suggested a stronger type I interferon response in COVID-19 ${ }^{18}$.

In the NK cells and (to a lesser extent) non-classical monocytes of patients with COVID-19, we found remarkably high expression of interferon-induced transmembrane protein 3 (IFITM3). IFITM3 plays an essential role in restricting viral replication in CAP-flu ${ }^{49}$, highlighted in a seminal paper demonstrating that, when infected with an influenza virus of limited virulence, mice lacking IFITM3 developed severe pulmonary inflammation ${ }^{50}$. The exact role of IFITM3 in COVID-19 still needs to be elucidated, but a recent study found explicit upregulation of IFITM3 in SARS-CoV-2 infected epithelial cells ${ }^{51}$, and a polymorphism of IFITM3 has been associated with disease severity and hospitalization ${ }^{52,53}$. Our results underline a potential role of IFITM3 in COVID-19, although mechanistic evidence will be decisive. 
Immune suppression is a hallmark of the immune dysregulation that characterizes sepsis ${ }^{28,29}$. Interestingly, we found a pronounced reduction of HLA-DR expression on classical monocytes in CAP-flu when compared with controls at both the transcriptional and the protein level. When directly comparing the three disease groups, HLA-DR protein expression on classical monocytes in COVID-19 was on par with CAP-flu, and both were lower than the expression in CAP-other. Immune suppression, as part of a severely dysregulated host response, has been suggested to play a role in COVID-19 ${ }^{54}$. Schulte-Schrepping et al. demonstrated a predominance of activated monocytes with a type I interferon signature and HLA-DR ${ }^{\text {high }}$ expression early in mild disease, whereas in severe disease was associated with persistent and dysfunctional HLA-DR ${ }^{\text {low }}$ monocytes ${ }^{9}$. HLA-DR expression on monocytes has been reported to progressively decrease as disease severity increases ${ }^{3,55}$. Kox et al also reported low monocyte HLA-DR expression in critically ill COVID-19 patients when compared with healthy controls, but this effect - and by implication the level of immune suppression - was modest when compared with bacterial septic shock ${ }^{56}$. In our cohort, HLA-DR surface protein expression was only reduced on monocytes of patients with viral pneumonia.

Strengths of this study include the application of the novel CITE-seq technique, directly integrating proteomic surface marker and transcriptomic data on a single-cell level. This enabled us to readily validate transcriptome-derived clusters and certain disease-related transcriptional patterns at the protein level. Furthermore, the design of this study (in terms of matching and sampling within 48 hours after hospital admission) and the inclusion of disease controls strengthen the observations. Our investigation has several limitations. Experimentally, the total number of analyzed cells was at the lower end of standard practice, which was mainly due to our multiplexed and integrated design. Differences in patient characteristics, such as body mass index and duration of symptoms prior to admission, were largely representative of 
expected differences between the patient populations ${ }^{5}$. The causative organism in CAP is typically only identified in approximately $50 \%$ of patients ${ }^{57,58}$. While in this respect our (nonCOVID-19) CAP cohort is representative of a general CAP population, patients with CAPother likely are a more heterogeneous group than patients with a pneumonia caused by one pathogen. Finally, one subject in our cohort was co-infected with both Influenza A and $S$. pneumoniae. We nevertheless decided to include this patient to create a realistic representation of patients with CAP-flu, as up to $30 \%$ of laboratory-confirmed pneumonia cases can involve viral and bacterial co-infections (most commonly Influenza and S. pneumoniae) ${ }^{58,59}$.

Collectively, this investigation provides insight into the peripheral immune features of CAP, including COVID-19, at the single-cell level in patients admitted to a general hospital ward. By contrasting multiple commonly encountered forms of CAP in a matched design that limits the influence of major confounding factors, we provide a framework for the roles of T cells, NK cells and monocytes in the immunopathophysiology of CAP. This knowledge could guide future mechanistic studies seeking pathogen-specific interventions. 


\section{Methods}

\section{Subjects and sample collection}

All individual subject data, such as age, sex and comorbidities can be found in Supplementary Table 1. This study was part of the ELDER-BIOME project (clinicaltrials.gov identifier NCT02928367) approved by the medical ethical committee of the Amsterdam UMC location AMC. Written informed consent was obtained from all participants or their legal representatives. In the context of the ELDER-BIOME project, trained research physicians screened patients older than 18 years admitted between October 2018 and June 2020 to the Amsterdam UMC, Flevohospital or BovenIJ hospital in the Netherlands. Patients were included if they were admitted to the ward and met all of the following criteria: clinical suspicion of an acute infection of the respiratory tract, defined as the presence of least one respiratory symptom (new cough or sputum production, chest pain, dyspnea, tachypnea, abnormal lung examination, or respiratory failure) and one systemic symptom (documented fever or hypothermia, leukocytosis or leukopenia), and an evident new or progressive infiltrate, consolidation or pleural effusion on chest X ray or computed tomography scan. Patients were excluded if there was a clinical suspicion of aspiration pneumonia or hospitalassociated pneumonia, or if community-acquired pneumonia was not the main reason for admission. Patients were excluded from this study if they were severely immunocompromised by either disease or medication. All COVID-19 patients had reverse transcription (RT-PCR)confirmed SARS-CoV-2 infection, in combination with a CORADS CT-score of $5^{60}$. Heparin anticoagulated blood was obtained within 48 hours of hospital admission. Age and sexmatched subjects without an infection were included as controls. 
For all patients and controls, heparin anticoagulated whole-blood was processed within 4 hours of sampling. PBMCs were separated by density gradient centrifugation using Ficoll-Paque Plus medium (GE Healthcare Life science, Little Chalfont, UK) and washed twice, first with cold PBS and then with cold PBS supplemented with $0.5 \%$ sterile endotoxin-free bovine serum albumin (BSA) (Divbio Science Europe, Breda, the Netherlands). PBMCs were resuspended in PBS containing $0.5 \%$ BSA and $2 \mathrm{mM}$ EDTA and the number of PBMCs was determined using a Coulter Counter (Beckman Coulter, Woerden, Netherlands). PBMCs were resuspended in IMDM medium containing 20\% filter-sterilized fetal calf serum and pen/strep, after which an equal part of the same medium containing $20 \%$ DMSO was slowly added while continuously stirring and working on ice. 3-5 million PBMCs were viably stored in $1.8 \mathrm{ml}$ cryogenic vials (Corning \#430388), which were slowly brought to $-80^{\circ} \mathrm{C}$. After $24-72$ hours, the cells were transferred to liquid nitrogen storage until further analysis.

\section{Sorting and staining}

After PBMCs were thawed, Fixable Viability Dye kit (eBioscience, San Diego, CA, USA) was used to assess cell viability by FACS analysis ( $>90 \%$ in all samples). 500,000 viable singletevents were sorted per sample using a Sony SH800 Cell Sorter (Sony Biotechnology, San Jose, CA, USA). The sorted-cells were incubated with Fc blocker (CD16/CD32, eBiosciences) for 10 minutes, after which each sample was incubated with TotalSeq Hashtag antibody tags to enable multiplexing and subsequent deconvoluting. After Hashtag staining the cells were pooled into four pools of five samples, each sample contributing equally to their respective pool. Cells were counted manually by light microscopy and Neubauer chambers. Each pooled sample was then incubated for 30 minutes with our TotalSeq oligo-conjugated antibody panel for later surface protein marker quantification. 


\section{Single-cell library generation}

Libraries were generated using the Chromium Single Cell 5' Library \& Gel Bead Kit v1.1 (10X genomics, Pleasanton, CA) following the manufacturer's instructions. In short, pooled cells were loaded aiming the capture of 10.000 cells per pool. Libraries were generated according to standard protocol (Chromium Next GEM Single Cell V(D)J Reagent Kits v1.1 rev E). The amplified mRNA and antibody-derived tags were divided by size and sequenced separately.

\section{Sequencing}

Samples were sequenced using a Hiseq4000 150PE mode. Each position from the 10x chip was loaded into 1 HiSeq 4000 lane. All the four antibody derived libraries were pooled together and sequenced in 1 HiSeq4000 lane.

$m R N A$, hashtags and antibody alignment

All the libraries were aligned using CellRanger 3.1 (10x genomics). For the hashtags and antibody tags we inputted their sequences as provided by Biolegend (San Diego, California, USA) and tag structure as informed on the Cellranger website.

\section{Cell deconvolution}

To deconvolute the cells belonging to each sample we used the R package Seurat (v3) ${ }^{61}$. The outputs derived from CellRanger were used to create two separate objects (one with the transcriptome alignment and one with the antibody plus hashtags (HTO) alignment). Initial objects were created using the function "Read10X". We filtered both objects based on the cell barcode to keep only cells which were identified in both the transcriptome and in the antibody 
alignments. After this cell filtering, we used the function "CreateSeuratObject" to create a transcriptome-based Seurat object. The antibody derived data was filtered to maintain only the hashtag counts; later it was appended as a specific assay using the "CreateAssayObject" function. Antibody reads were normalized using the CLR method. For cell demultiplexing we used the function "HTODemux" with the parameters "init=9", "nsamples=10000" and "positivie quantile" ranging between 0.999 and 0.9999999 per each sample, in order to maximize the number of singlets detected. Individual single cells were finally filtered based on their assigned "HTO_classification.global"= "Singlet".

\section{Antibody quantification and normalization}

Antibody data was normalized using the Seurat function "NormalizeData"with the parameters "normalization.method" = "CLR" and "margin"="2", to indicate a normalization across cells.

\section{Quality control}

Cells were filtered based on number of features (nfeature_RNA), number of genes (nCount_RNA) and percentage of mitochondrial reads (percent.mt) using Seurat “subset(object, subset $=$ nCount_RNA $>1000$ \&nCount_RNA $<10000 \&$ nFeature_RNA $>$ $200 \&$ percent.mt $<20$ )".

\section{Data scaling, normalization and cell cycle correction}

We calculated cell cycle scores as following ("CellCycleScoring(Healthy_object_Cycle, s.features $=$ s.genes, g2m.features $=\mathrm{g} 2 \mathrm{~m}$.genes, set.ident $=$ TRUE") using a list of S phase and G2M phase genes preloaded in Seurat. Cells were then scaled and normalized using the function "SCTransform(object, $\quad$ vars.to.regress = c("nCount_RNA","percent.mt","S.Score", "G2M.Score"))" 


\section{Clustering and visualization}

Principal components for each set of cells as shown in individual Figures were identified using the "RunPCA" function. Cells were then further processed for clustering and visualization using the Seurat functions "FindNeighbors", "FindClusters"and "RunUMAP". The number of principal components used as input were determined by using the "ElbowPlot" function and identifying the number of the PCs which explain most of the data variance.

\section{Differential expression analysis}

Differential expression analysis was performed using the functions "FindAllMarkers" or "FindMarkers" and the following parameters: "min.pct $=0.25$, logfc.threshold $=0.25$, assay="SCT"'”.

\section{List of software used}

CellRanger 3.1; R version 3.6.3 (2020-02-29), FlowJo V10.7, the R packages: Seurat v3, corrplot, dplyr, Matrix, cowplot, rstatix, ggplot2; Adobe Illustrator 2021.

\section{Quantification and statistical analysis}

All statistical analyses were performed using R version 3.6.3. Enrichment analysis were done by first generating contingency tables with the cell distributions per cluster. Chi-squares for the contingency tables were calculated ("chisq <- chisq.test(table)", the residuals rounded "round(chisq\$residuals, 4)" and correlation plots generated "corrplot(chisq\$residuals, is.cor = FALSE, $\quad \operatorname{col}=\operatorname{rev}($ brewer.pal $(n=8, \quad$ name $=" R d B u ")), \quad$ tl.cex $=1, \quad$ cl.pos $=" r ", \quad$ cl.ratio=1, cl.length=10)" with the graphical parameters shown adjusted per cluster. Comparisons with more than two residuals of difference at least were considered biologically relevant. In the box 
and whisker plots data was represented with a median line and a box indicating the interquartile range, with individual data points shown (Figs. 1h, 2h, and 4h). Significance was defined as $\mathrm{p}$ $<0.05$. Statistical significance was determined using either the two-sided Wilcoxon rank-sum test (Fig. 1h) or the two-sided Kruskal-Wallis test with post-hoc pairwise Dunn's test with Benjamini-Hochberg p-value adjustment (Figs. $2 \mathrm{~h}$ and $4 \mathrm{~h}$ ). All gene expression analyses were corrected for multiple testing using the Benjamini-Hochberg method, with significance defined as an adjusted $\mathrm{p}<0.05$. 


\section{References}

1. Huang, C. et al. Clinical features of patients infected with 2019 novel coronavirus in Wuhan, China. Lancet 395, 497-506 (2020).

2. Richardson, S. et al. Presenting Characteristics, Comorbidities, and Outcomes Among 5700 Patients Hospitalized With COVID-19 in the New York City Area. JAMA 323, $2052(2020)$.

3. Giamarellos-Bourboulis, E. J. et al. Complex Immune Dysregulation in COVID-19 Patients with Severe Respiratory Failure. Cell Host Microbe 1-9 (2020) doi:10.1016/j.chom.2020.04.009.

4. Weinberger, D. M. et al. Estimation of Excess Deaths Associated With the COVID-19 Pandemic in the United States, March to May 2020. JAMA Intern. Med. 180, 1336$1344(2020)$.

5. Wiersinga, W. J., Rhodes, A., Cheng, A. C., Peacock, S. J. \& Prescott, H. C. Pathophysiology, Transmission, Diagnosis, and Treatment of Coronavirus Disease 2019 (COVID-19): A Review. JAMA - J. Am. Med. Assoc. 2019, 1-13 (2020).

6. Zheng, Y. et al. Inflammatory responses relate to distinct bronchoalveolar lavage lipidome in community-acquired pneumonia patients: A pilot study. Respir. Res. 20, 82 (2019).

7. Chen, Z. \& John Wherry, E. T cell responses in patients with COVID-19. Nat. Rev. Immunol. 20, 529-536 (2020).

8. De Biasi, S. et al. Marked T cell activation, senescence, exhaustion and skewing towards TH17 in patients with COVID-19 pneumonia. Nat. Commun. 11, 1-17 (2020).

9. Schulte-Schrepping, J., Reusch, N., Paclik, D., Saliba, A.-E. \& Sander, L. E. Severe 
COVID-19 Is Marked by a Dysregulated Myeloid Cell Compartment. Cell (2020) doi:10.1016/j.cell.2020.08.001.

10. Merad, M. \& Martin, J. C. Pathological inflammation in patients with COVID-19: a key role for monocytes and macrophages. Nat. Rev. Immunol. 2, (2020).

11. Zhang, J.-Y. et al. Single-cell landscape of immunological responses in patients with COVID-19. Nat. Immunol. 21, 1-12 (2020).

12. Lee, J. S. et al. Immunophenotyping of COVID-19 and influenza highlights the role of type I interferons in development of severe COVID-19. Sci. Immunol. 5, 1554 (2020).

13. Wilk, A. J. et al. A single-cell atlas of the peripheral immune response in patients with severe COVID-19. Nat. Med. 26, 1070-1076 (2020).

14. Kuri-Cervantes, L. et al. Immunologic perturbations in severe COVID-19/SARS-CoV2 infection. bioRxiv Prepr. Serv. Biol. (2020) doi:10.1101/2020.05.18.101717.

15. Stolk, R. F. et al. Potentially inadvertent immunomodulation: Norepinephrine use in sepsis. American Journal of Respiratory and Critical Care Medicine vol. 194 550-558 (2016).

16. Van Der Poll, T., Van De Veerdonk, F. L., Scicluna, B. P. \& Netea, M. G. The immunopathology of sepsis and potential therapeutic targets. Nat. Rev. Immunol. 17, 407-420 (2017).

17. Su, Y. et al. Multi-Omics Resolves a Sharp Disease-State Shift between Mild and Moderate COVID-19. Cell 183, 1479-1495.e20 (2020).

18. Zhu, L. et al. Single-Cell Sequencing of Peripheral Mononuclear Cells Reveals Distinct Immune Response Landscapes of COVID-19 and Influenza Patients. Immunity 53, 685-696.e3 (2020). 
19. Stoeckius, M. et al. Simultaneous epitope and transcriptome measurement in single cells. Nat. Methods 14, 865-868 (2017).

20. Subbe, C. P., Kruger, M., Rutherford, P. \& Gemmel, L. Validation of a modified early warning score in medical admissions. QJM - Mon. J. Assoc. Physicians 94, 521-526 (2001).

21. Churpek, M. M. et al. Quick Sepsis-related Organ Failure Assessment, Systemic Inflammatory Response Syndrome, and Early Warning Scores for Detecting Clinical Deterioration in Infected Patients outside the Intensive Care Unit. Am. J. Respir. Crit. Care Med. 195, 906-911 (2017).

22. Martin, M. D. \& Badovinac, V. P. Defining memory CD8 T cell. Frontiers in Immunology vol. 9 (2018).

23. Nicolet, B. P. et al. CD29 identifies IFN- $\gamma$-producing human CD8+ T cells with an increased cytotoxic potential. Proc. Natl. Acad. Sci. U. S. A. 117, 6686-6696 (2020).

24. Sampath, P., Moideen, K., Ranganathan, U. D. \& Bethunaickan, R. Monocyte Subsets: Phenotypes and Function in Tuberculosis Infection. Frontiers in immunology vol. 9 $1726(2018)$.

25. Pang, Z., Raudonis, R., McCormick, C. \& Cheng, Z. Early growth response 1 deficiency protects the host against pseudomonas aeruginosa lung infection. Infect. Immun. 88, (2020).

26. Zannas, A. S. et al. Epigenetic upregulation of FKBP5 by aging and stress contributes to NF-кB-driven inflammation and cardiovascular risk. Proc. Natl. Acad. Sci. U. S. A. 166, 11370-11379 (2019).

27. Zaiss, D. M. W., Gause, W. C., Osborne, L. C. \& Artis, D. Emerging functions of 
amphiregulin in orchestrating immunity, inflammation, and tissue repair. Immunity vol. $42216-226(2015)$.

28. Venet, F. \& Monneret, G. Advances in the understanding and treatment of sepsisinduced immunosuppression. Nat. Rev. Nephrol. 14, 121-137 (2018).

29. Hotchkiss, R. S., Monneret, G. \& Payen, D. Sepsis-induced immunosuppression: From cellular dysfunctions to immunotherapy. Nat. Rev. Immunol. 13, 862-874 (2013).

30. Verhelst, J., Parthoens, E., Schepens, B., Fiers, W. \& Saelens, X. Interferon-Inducible Protein Mx1 Inhibits Influenza Virus by Interfering with Functional Viral Ribonucleoprotein Complex Assembly. J. Virol. 86, 13445-13455 (2012).

31. Sharma, S. et al. APOBEC3A cytidine deaminase induces RNA editing in monocytes and macrophages. Nat. Commun. 6, (2015).

32. Verma, K. et al. Human CD8+ CD57- TEMRA cells: Too young to be called 'old'. PLoS One 12, (2017).

33. Schmidt, M. E. \& Varga, S. M. The CD8 T Cell Response to Respiratory Virus Infections. Front. Immunol. 9, 678 (2018).

34. Mathew, D. et al. Deep immune profiling of COVID-19 patients reveals patient heterogeneity and distinct immunotypes with implications for therapeutic interventions. bioRxiv Prepr. Serv. Biol. (2020) doi:10.1101/2020.05.20.106401.

35. Kuri-Cervantes, L. et al. Comprehensive mapping of immune perturbations associated with severe COVID-19. Sci. Immunol. 5, 1-20 (2020).

36. Neidleman, J. et al. SARS-CoV-2-Specific T Cells Exhibit Phenotypic Features of Helper Function, Lack of Terminal Differentiation, and High Proliferation Potential. Cell Reports Med. 1, 100081 (2020). 
37. Weiskopf, D. et al. Phenotype and kinetics of SARS-CoV-2-specific T cells in COVID-19 patients with acute respiratory distress syndrome. Sci. Immunol. 5, 1-15 (2020).

38. Liao, M. et al. Single-cell landscape of bronchoalveolar immune cells in patients with COVID-19. Nat. Med. 26, 842-844 (2020).

39. Laing, A. G. et al. A dynamic COVID-19 immune signature includes associations with poor prognosis. Nat. Med. (2020) doi:10.1038/s41591-020-1038-6.

40. Market, M. et al. Flattening the COVID-19 Curve With Natural Killer Cell Based Immunotherapies. Frontiers in Immunology vol. 11 (2020).

41. Fox, A. et al. Severe Pandemic H1N1 2009 Infection Is Associated with Transient NK and T Deficiency and Aberrant CD8 Responses. PLoS One 7, e31535 (2012).

42. Jost, S. et al. Changes in Cytokine Levels and NK Cell Activation Associated with Influenza. PLoS One 6, e25060 (2011).

43. Marcken, M. de, Dhaliwal, K., Danielsen, A. C., Gautron, A. S. \& Dominguez-Villar, M. TLR7 and TLR8 activate distinct pathways in monocytes during RNA virus infection. Sci. Signal. 12, 1347 (2019).

44. García-Sastre, A. Induction and evasion of type I interferon responses by influenza viruses. Virus Research vol. 162 12-18 (2011).

45. Walsh, D. \& Mohr, I. Viral subversion of the host protein synthesis machinery. Nature Reviews Microbiology vol. 9 860-875 (2011).

46. Chen, X. et al. Host immune response to influenza A virus infection. Frontiers in Immunology vol. 9 (2018). 
47. Hoeve, M. A., Nash, A. A., Jackson, D., Randall, R. E. \& Dransfield, I. Influenza virus A infection of human monocyte and macrophage subpopulations reveals increased susceptibility associated with cell differentiation. PLoS One 7, (2012).

48. Pontelli, M. C. et al. 3 Divisions of Clinical Immunology; 154 Infectious Diseases, 5 Intensive Care Unit; 6 Department of Cell and 16 Molecular Biology and Pathogenic Bioagents. bioRxiv 7, 2020.07.28.225912 (2020).

49. Yánez, D. C., Ross, S. \& Crompton, T. The IFITM protein family in adaptive immunity. Immunology 159, 365-372 (2020).

50. Everitt, A. R. et al. IFITM3 restricts the morbidity and mortality associated with influenza. Nature 484, 519-523 (2012).

51. Hachim, M. Y. et al. Interferon-Induced Transmembrane Protein (IFITM3) Is Upregulated Explicitly in SARS-CoV-2 Infected Lung Epithelial Cells. Front. Immunol. 11, (2020).

52. Jin, R. et al. The Journal of Infectious Diseases Interferon-Induced Transmembrane Protein 3 Genetic Variant rs12252-C Associated With Disease Severity in Coronavirus Disease 2019. J. Infect. Dis. ${ }^{\circledR}$ 222, 34-41 (2020).

53. Gómez, J. et al. The Interferon-induced transmembrane protein 3 gene (IFITM3) rs $12252 \mathrm{C}$ variant is associated with COVID-19. Cytokine 137, 155354 (2020).

54. Remy, K. E. et al. Severe immunosuppression and not a cytokine storm characterizes COVID-19 infections. JCI insight 5, (2020).

55. Spinetti, T. et al. Reduced Monocytic Human Leukocyte Antigen-DR Expression Indicates Immunosuppression in Critically Ill COVID-19 Patients. Anesth. Analg. 131, 993-999 (2020). 
56. Kox, M. et al. COVID-19 patients exhibit less pronounced immune suppression compared with bacterial septic shock patients. Crit. Care 24, 4-7 (2020).

57. Welte, T., Torres, A. \& Nathwani, D. Clinical and economic burden of communityacquired pneumonia among adults in Europe. Thorax 67, 71-79 (2012).

58. Jain, S. et al. Community-Acquired Pneumonia Requiring Hospitalization among U.S. Adults. N. Engl. J. Med. 373, 415-427 (2015).

59. Gupta, R. K., George, R. \& Nguyen-Van-Tam, J. S. Bacterial Pneumonia and Pandemic Influenza Planning. Emerg. Infect. Dis. 14, 1187-1192 (2008).

60. De Smet, K. et al. Diagnostic Performance of Chest CT for SARS-CoV-2 Infection in Individuals with or without COVID-19 Symptoms. Radiology 202708 (2020) doi:10.1148/radiol.2020202708.

61. Stuart, T. et al. Comprehensive Integration of Single-Cell Data. Cell 177, 18881902.e21 (2019). 


\section{Acknowledgements}

Funding: This work was supported by the Corona Research Fund from the AMC Foundation. T.D.Y.R. and A.S. are supported by NACTAR (\# 16447) financed by the Dutch Research Council (NWO). J.B. and C.L. are supported by the European Commission (FAIR \# 847786). Author contributions: ARS \& TDYR; conceptualization, data curation, formal analysis, investigation, methodology, project administration, writing - original draft, writing - review \& editing. AS \& IRM; investigation, writing - review \& editing. MS \& JdB \& CL; investigation, data curation, writing - review \& editing. LV; funding acquisition, writing - review \& editing. BPS; funding acquisition, methodology, writing - review \& editing. WJW; funding acquisition, supervision, writing - review \& editing. FAVB; data curation, formal analysis, methodology, software, visualization, writing - review \& editing. TvdP; conceptualization, funding acquisition, methodology, resources, supervision, writing - review \& editing. Competing interests: The authors declare no competing interests. Data and materials availability: The datasets generated during this study are available at Gene Expression Omnibus (GEO) [accession code GSE164948].

\section{Figures and tables}


Table 1. Clinical characteristics and disease course.

\begin{tabular}{|c|c|c|}
\hline $\begin{array}{l}\text { COVID-19 } \\
(n=8)\end{array}$ & $\begin{array}{l}\text { CAP }^{1} \\
(n=8)\end{array}$ & Controls $(n=4)$ \\
\hline
\end{tabular}

\begin{tabular}{|c|c|c|c|c|}
\hline \multicolumn{5}{|l|}{ DEMOGRAPHICS } \\
\hline Age (years) & $66.9(9.4)$ & $70.9(14.3)$ & $72.2(1.7)$ & 0.35 \\
\hline Sex (male) & 5 & 5 & 2 & $>0.99$ \\
\hline Body mass index & $32.8(6.5)$ & $23.8(7.6)$ & $25.7(4.9)$ & 0.08 \\
\hline Race (white/black) & $4 / 4$ & $7 / 1$ & $4 / 0$ & 0.19 \\
\hline \multicolumn{5}{|l|}{ CHRONIC COMORBIDITIES } \\
\hline COPD & 0 & 3 & 0 & 0.19 \\
\hline Asthma & 0 & 2 & 0 & 0.33 \\
\hline Hypertension & 4 & 3 & 1 & 0.85 \\
\hline History of myocardial infarction & 0 & 2 & 0 & 0.33 \\
\hline History of stroke & 1 & 0 & 1 & 0.66 \\
\hline Diabetes mellitus, type 2 & 3 & 1 & 1 & 0.80 \\
\hline Chronic kidney disease & 0 & 1 & 1 & 0.66 \\
\hline \multicolumn{5}{|l|}{ CHRONIC MEDICATIONS } \\
\hline Inhaled corticosteroids & 0 & 2 & 0 & 0.33 \\
\hline Low-dose oral corticosteroids ${ }^{2}$ & 0 & 1 & 0 & $>0.99$ \\
\hline ACE-inhibitor/AT-II antagonist & 4 & 3 & 1 & 0.85 \\
\hline Statins & 3 & 1 & 3 & 0.16 \\
\hline Platelet aggregation inhibitors & 2 & 1 & 2 & 0.36 \\
\hline \multicolumn{5}{|l|}{ LABORATORY TESTS } \\
\hline Platelets $\left(\times 10^{9} / \mathrm{L}\right)$ & $284(112)$ & $294(85)$ & & 0.84 \\
\hline Leukocytes $\left(\times 10^{9} / \mathrm{L}\right)$ & $5.7[2.6,7.2]$ & $13.9[6.0,19.5]$ & & $<0.01$ \\
\hline Neutrophils (x109/L) & $4.4[1.5,5.8]$ & $11.7[5.3,17.9]$ & & $<0.01$ \\
\hline Lymphocytes (x109/L) & $0.8[0.4,1.5]$ & $1.1[0.5,3.1]$ & & 0.49 \\
\hline \multicolumn{5}{|l|}{ SEVERITY SCORES ${ }^{3}$} \\
\hline Modified Early Warning Score & $3.5[1.0,5.0]$ & $3.5[2.0,6.0]$ & & 0.87 \\
\hline Pneumonia Severity Index & $3.0[2.0,4.0]$ & $3.5[1.0,5.0]$ & & 0.23 \\
\hline CURB-65 & $1.0[0.0,2.0]$ & $1.0[0.0,3.0]$ & & 0.65 \\
\hline qSOFA & $1.0[0.0,1.0]$ & $1.0[0.0,1.0]$ & & $>0.99$ \\
\hline \multicolumn{5}{|l|}{ DISEASE COURSE } \\
\hline Symptoms to admission (days) & $10.0[2.0,14.0]$ & $3.5[2.0,9.0]$ & & 0.01 \\
\hline Hospital length of stay (days) & $3.5[1.0,6.0]$ & $3.0[2.0,8.0]$ & & 0.79 \\
\hline 28-day mortality & 1 & 0 & & $>0.99$ \\
\hline
\end{tabular}

$\mathrm{ACE}=$ angiotensin-converting enzyme; AT-II = angiotensin II; CAP = community-acquired pneumonia; CURB-65 = confusion, blood urea nitrogen, respiratory rate, blood pressure, age 65 or older; COPD = chronic obstructive pulmonary disease; qSOFA = quick sequential organ failure assessment score.

Continuous data are presented as mean (standard deviation) or median [range], and compared using a two-sided one-way analysis of variance (equal variances not assumed) or two-sided Kruskal-Wallis test, respectively. Categorical data are presented as counts and compared using Fisher's exact test.

${ }^{1}$ Caused by either Influenza A, bacterial or unknown pathogens

${ }^{2}$ Corticosteroids $<7.5 \mathrm{mg}$ prednisolone/day

${ }^{3}$ Measured upon presentation to the emergency department 

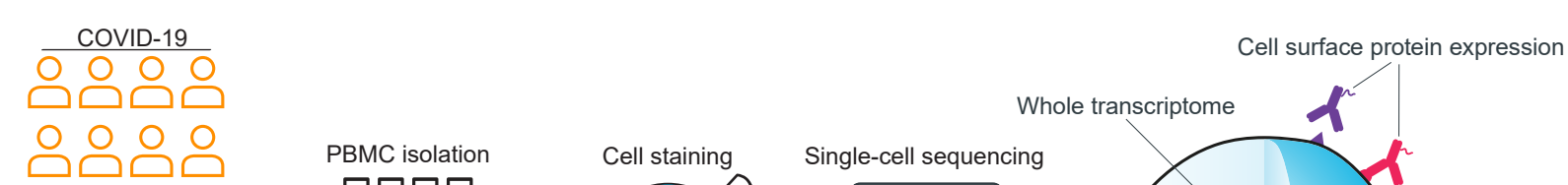

PBMC isolation

Whole transcriptome

CAP-other \& CAP-flu

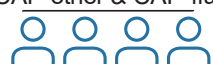

ํํํํำ

Controls

员员前员

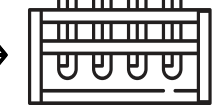

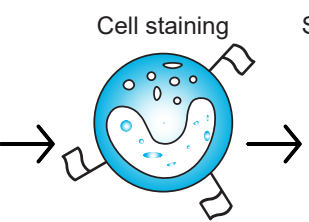

욤ำ

B
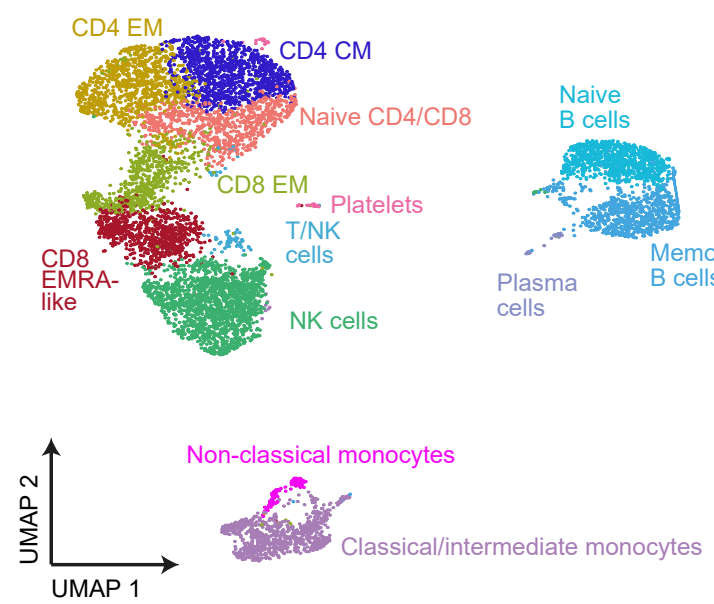

E

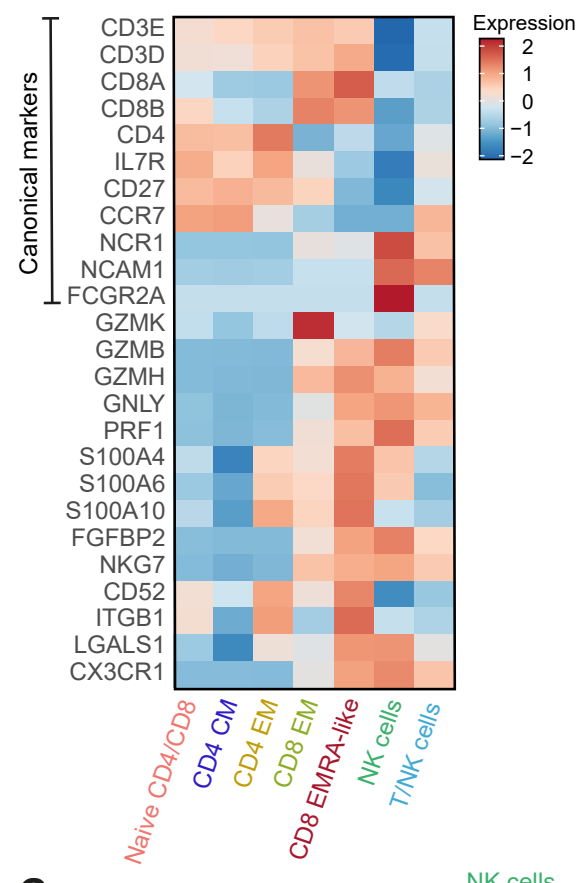

G

Type I interferon signaling pathway -

Endoplasmic reticulum membrane ER to Golgi transport -

MHC class II protein complex _ Interferon-gamma-mediated signaling Negative regulation of viral replication Peptide antigen binding -

$$
\text { Cell surface - }
$$

Antigen processing and presentation -

Antigen processing via MHC class I _

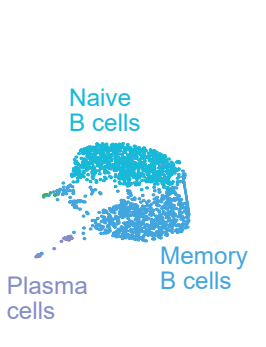

C
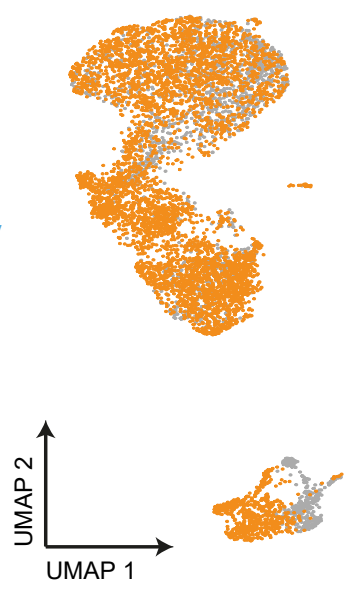

$\mathbf{F}$

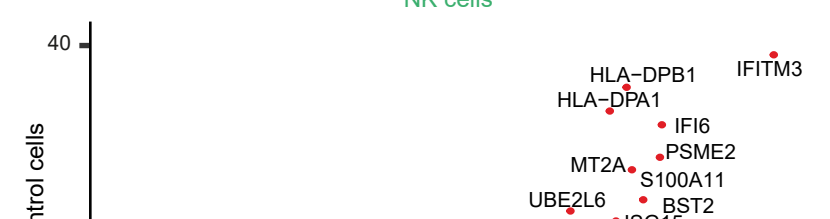

CARD16 16

ISG2O HSP9OAB1 LGALS1

TMBIM6 ALOXSAP1

PSMB9 TCF25 $\mathrm{CCL} 3$

RAC2 2 'PSBME1

CALR $\triangle \mathrm{CCL} 55^{\circ L Y 6 E}$

RARRES $3^{\circ} \mathrm{GZMH}$

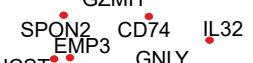

MT-ATP6

${ }_{\text {HCST }}{ }^{\circ}$ GZ3 GNMA

D

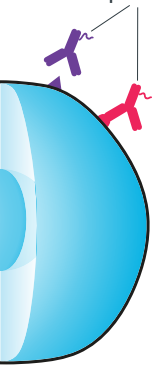


Fig. 1. The immune response in COVID-19 is characterized by an expansion of CD8 EMRA-like T cells and type I interferon-stimulated NK cells, both demonstrating high cytotoxic potential. (a) Experimental overview: PBMCs from a matched cohort of hospitalized patients with CAP caused by SARS-CoV-2 (COVID-19), CAP caused by Influenza A or other pathogens, and non-infectious controls, were isolated and stained with a panel of oligonucleotide-tagged antibodies. Single-cell mRNA and surface protein expression were subsequently measured on a 10X Genomics platform. (b,c) UMAPs depicting the clusters identified by the single-cell transcriptomic analysis of PBMCs from control subjects and patients with COVID-19, each dot representing a single cell. In the first UMAP (b) cells are colored by cell type cluster, whereas in the second UMAP (c) cells are colored by donor group. See also Supplementary Fig. 1. (d) Correlation plot depicting cluster enrichment in controls and COVID-19 patients. Dot size proportional to Pearson's residual of the chi-squared test (i.e., reflecting the difference between the observed and expected proportion), while the color represents the degree of association from Pearson's chi-squared residuals (red means a positive association, blue means a negative association). (e) Heatmap showing the expression of canonical genes and the top differentially expressed genes (DEGs) derived from comparing the CD8 EM and CD8 EMRA-like cell clusters (adjusted $\mathrm{p}<0.05$ ). The heatmap also shows the expression of these genes in the other identified T and NK cell clusters. See also Supplementary Fig. 2. (f) Graph depicting the DEGs identified when comparing cells from COVID-19 patients and controls within the NK cell cluster. The X-axis depicts the average log fold change and the $\mathrm{Y}$-axis depicts the percent point difference between the proportion of cells expressing the gene in the COVID-19 group minus the proportion of cells expressing the gene in the control group. All depicted DEGs are statistically significant after adjusting for multiple testing (BenjaminiHochberg). (g) Bar plot showing Gene Ontology pathway analysis of genes upregulated in NK cells from patients with COVID-19 (relative to controls) identified in the analysis of panel G. 
$\mathrm{X}$-axis shows the Benjamini-Hochberg adjusted $-\log 10 \mathrm{p}$-value from the enrichment score analysis. (h) Box and whisker plots showing the enrichment of the type I interferon pathway in all cell subsets, split between COVID-19 patients and controls. The Y-axis depicts the enrichment score. Statistical significance was determined using the two-sided Wilcoxon ranksum test: ${ }^{*} \mathrm{p}<0.05, * * \mathrm{p}<0.01$. 
Fig. 2. The peripheral immune features of $T$ cells, NK cells and monocytes vary between

CAP-flu and CAP-other. (a,b) UMAPs depicting the clusters identified by the single-cell transcriptomic analysis of PBMCs from controls, CAP-flu and CAP-other patients, where each dot represents a single cell. In the first UMAP (a) cells are colored by cell type cluster, whereas in the second UMAP (b) cells are colored by donor group. See also Supplementary Fig. 3. (c) Correlation plot depicting cluster enrichment in controls, CAP-flu and CAP-other patients. Dot size proportional to Pearson's residual of the chi-squared test (i.e., reflecting the difference between the observed and expected proportion), while the color represents the degree of association from Pearson's chi-squared residuals (red means a positive association, blue means a negative association). (c) Heatmap showing the expression of canonical genes and the top differentially expressed genes (DEGs) derived from comparing the CD8 EM and CD8 EMRAlike cell clusters (adjusted $\mathrm{p}<0.05$ ). The heatmap also shows the expression of these genes in the other identified T and NK cell clusters. See also Supplementary Fig. 4. (e) Density plots showing the surface protein expression of CD38, CD33, CD11b and CD11c per myeloid cell cluster. (f) Graph depicting the DEGs in the classical monocyte cluster when comparing CAPflu patients versus controls. The $\mathrm{X}$-axis depicts the average log fold change and the Y-axis depicts the percent point difference between the proportion of cells expressing the gene in the CAP-flu group minus the proportion of cells expressing the gene in the control group. All depicted DEGs are statistically significant after adjusting for multiple testing (BenjaminiHochberg). (g) Bar plot showing Gene Ontology pathway analysis of downregulated genes identified in the analysis of panel G. X-axis shows the Benjamini-Hochberg adjusted -log10 pvalue from the enrichment score analysis. (h) Boxplots depicting the downregulation of the MHC class II protein complex transcriptional pathway in naive B cells, memory B cells, classical monocytes and non-classical monocytes clusters, split between controls, CAP-flu and CAP-other patients. Statistical significance was determined using the two-sided Kruskal-Wallis 
test with post-hoc pairwise Dunn's test: $*$ BH-adjusted $\mathrm{p}<0.05$. (i) Density plot showing the normalized surface protein expression of HLA-DR on cells in naive B cells, memory B cells, classical monocytes and non-classical monocytes clusters, split between controls, CAP-flu and CAP-other patients. 
A

B
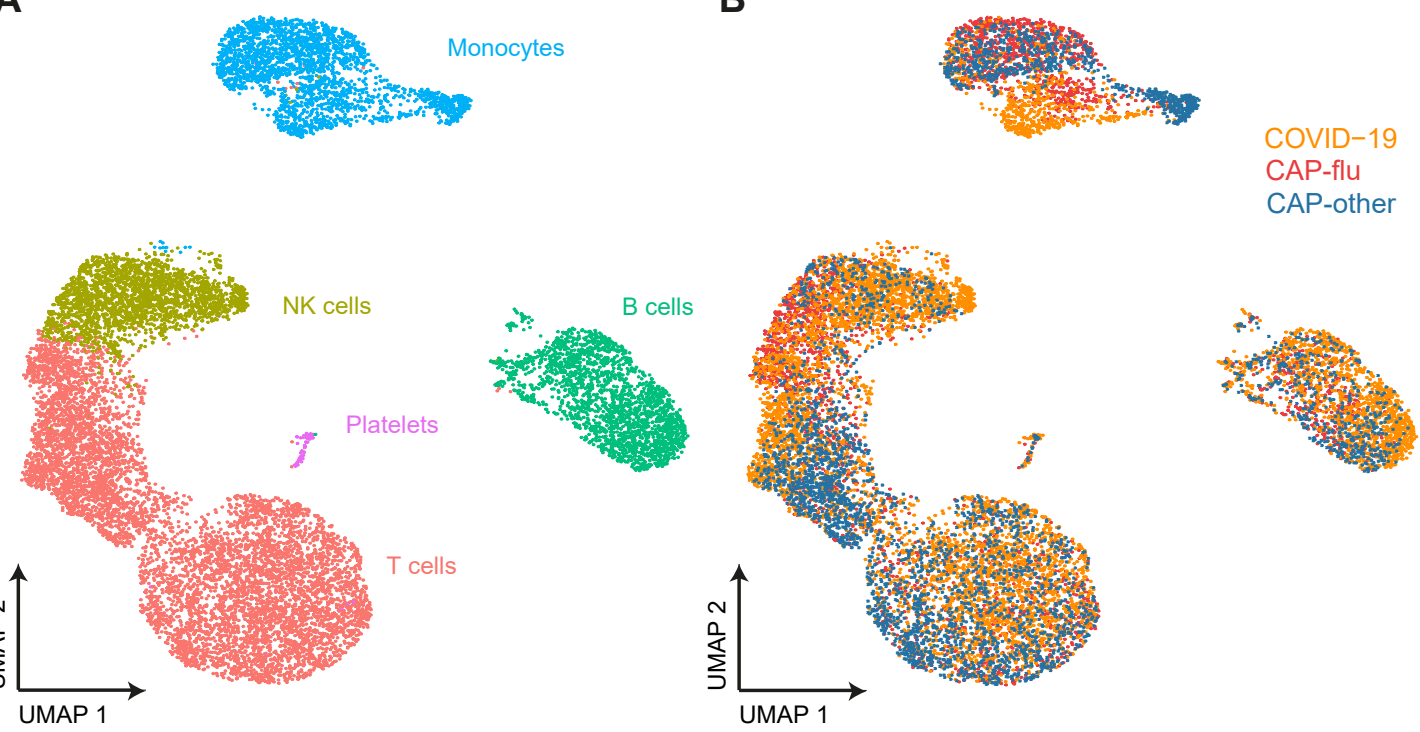

COVID-19

CAP-flu

CAP-other

$\sum_{3}^{2}$

D

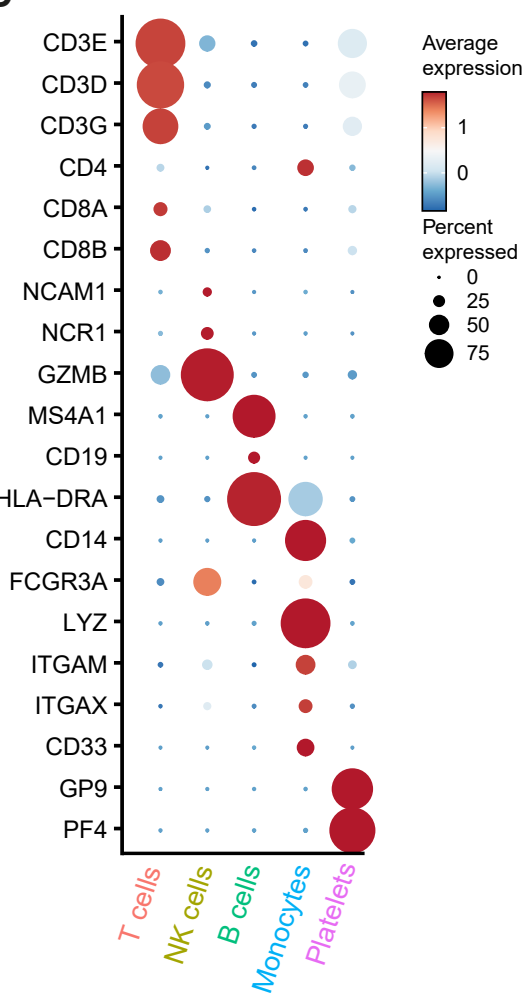

E

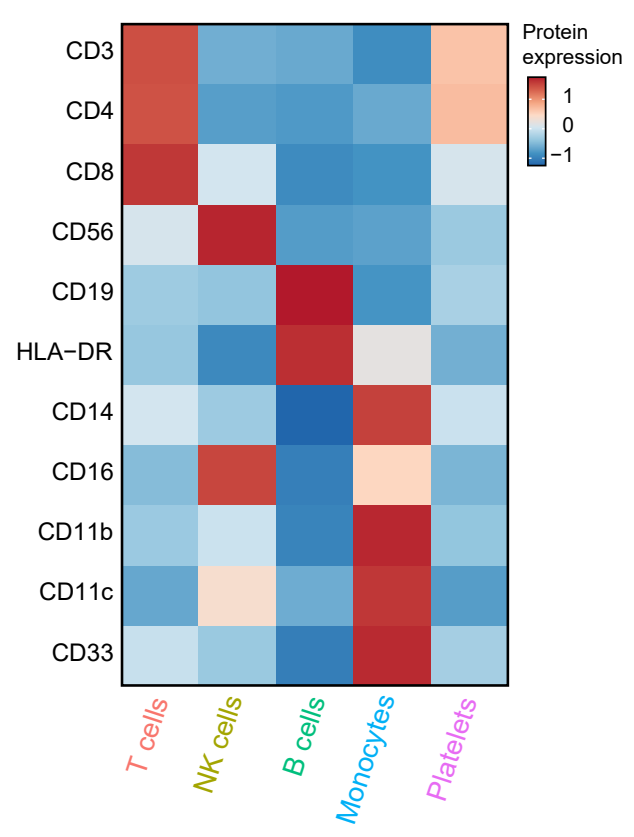

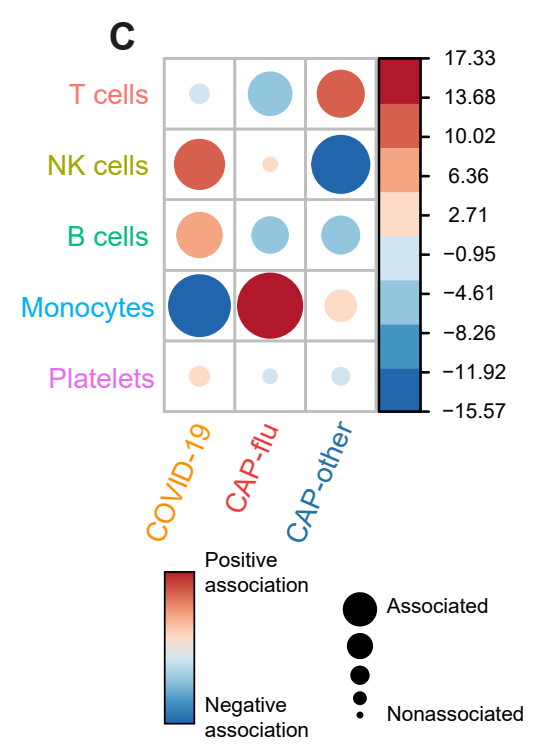

F

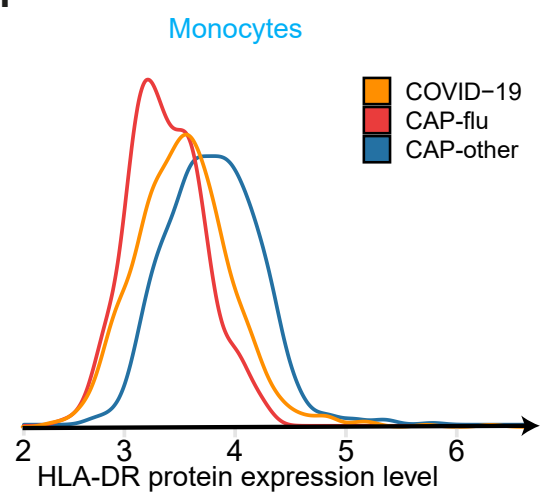


Fig. 3. Divergent composition of major immune cell types in patients with COVID-19, CAP-flu and CAP-other. (a,b) UMAPs depicting the metaclusters identified by the single-cell transcriptomic analysis of PBMCs from controls, COVID-19, CAP-flu and CAP-other patients, where each dot represents a single cell. In the first UMAP (a) cells are colored by cell type cluster, whereas in the second UMAP (b) cells are colored by donor group. (c) Correlation plot depicting metacluster enrichment in COVID-19, CAP-flu or CAP-other patients. Dot size proportional to Pearson's residual of the chi-squared test (i.e., reflecting the difference between the observed and expected proportion), while the color represents the degree of association from Pearson's chi-squared residuals (red means a positive association, blue means a negative association). (d) Dot plot showing canonical genes per identified metacluster. Color indicates the normalized level of expression, while the dot size is proportional to the percentage of cells per cluster expressing the canonical gene. (e) Heatmap showing the expression of lineagedefining protein surface markers per metacluster. (f) Density plot showing the normalized surface protein expression of HLA-DR on the monocyte lineage, split between COVID-19, CAP-flu and CAP-other patients. 
A

B

Granulysin+ NK cells

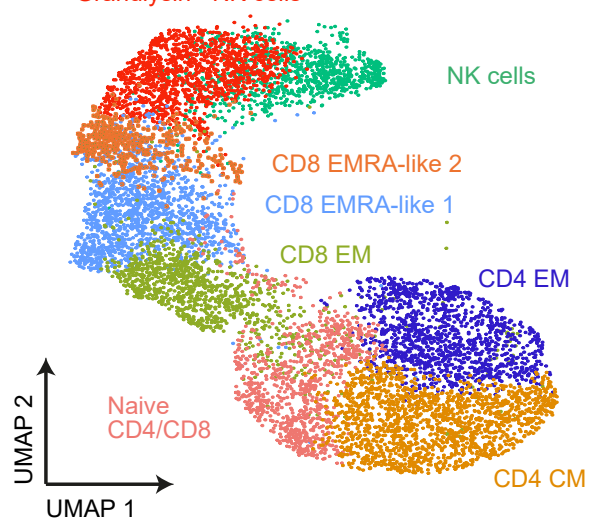

E

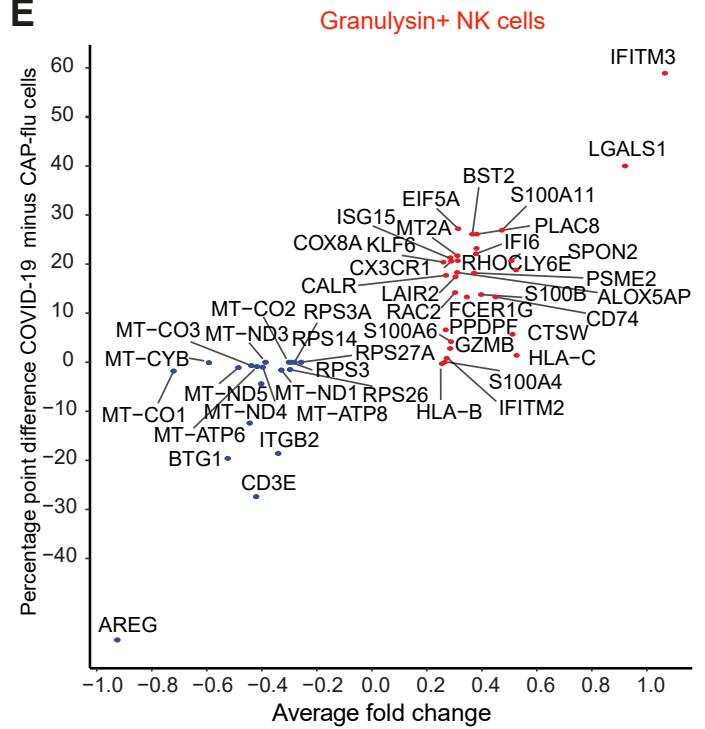

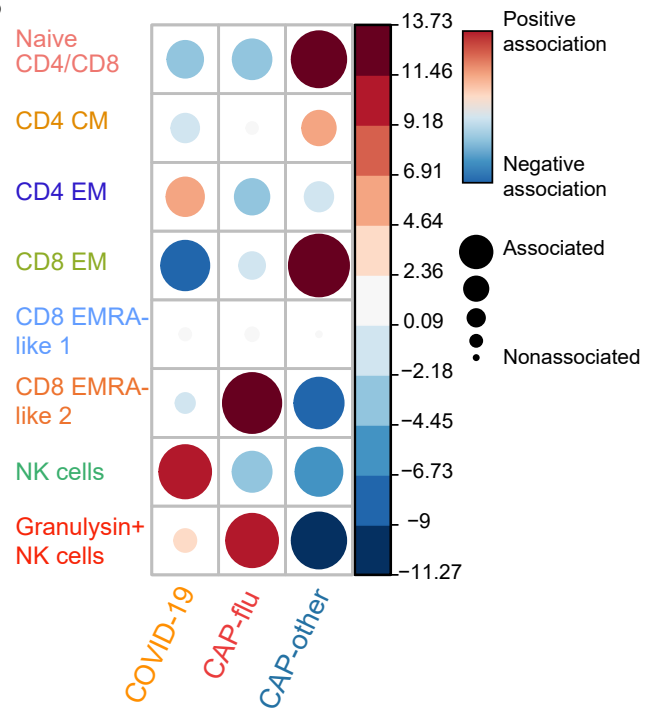

c

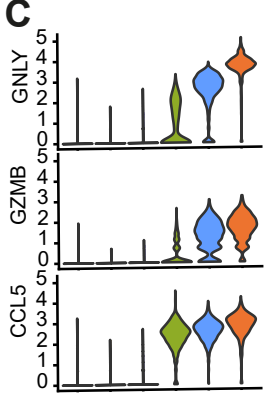

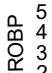

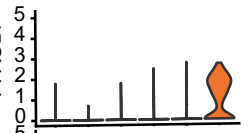

$\times 4$

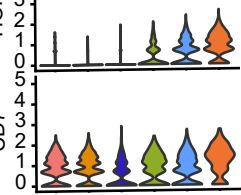

(1)

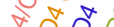

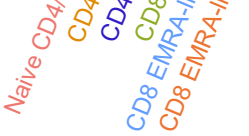

D

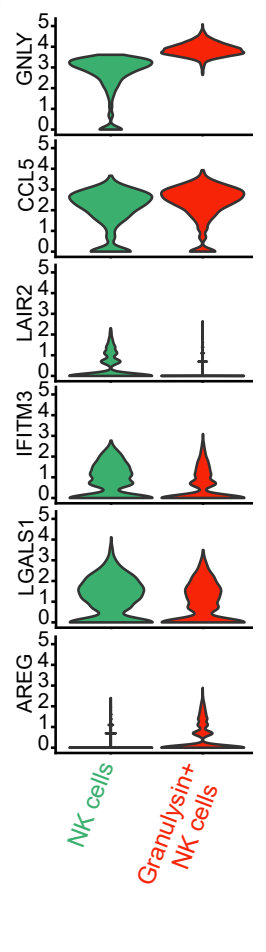

$\mathbf{F}$

Granulysin+ NK cells

Type I interferon signaling pathway -

Endoplasmic reticulum membrane -

Calcium-dependent protein binding Cell surface -

Regulation of viral genome replication -

S100 protein binding Immune response.

Response to interferon-beta -

Response to interferon-alpha Defense response to virus -

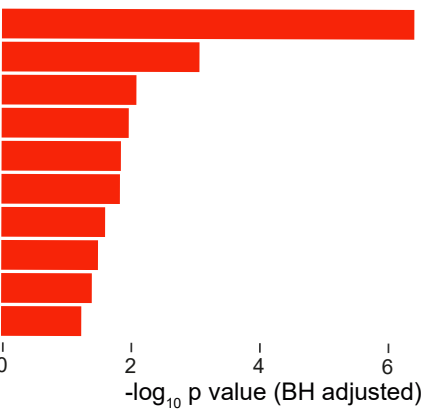

H

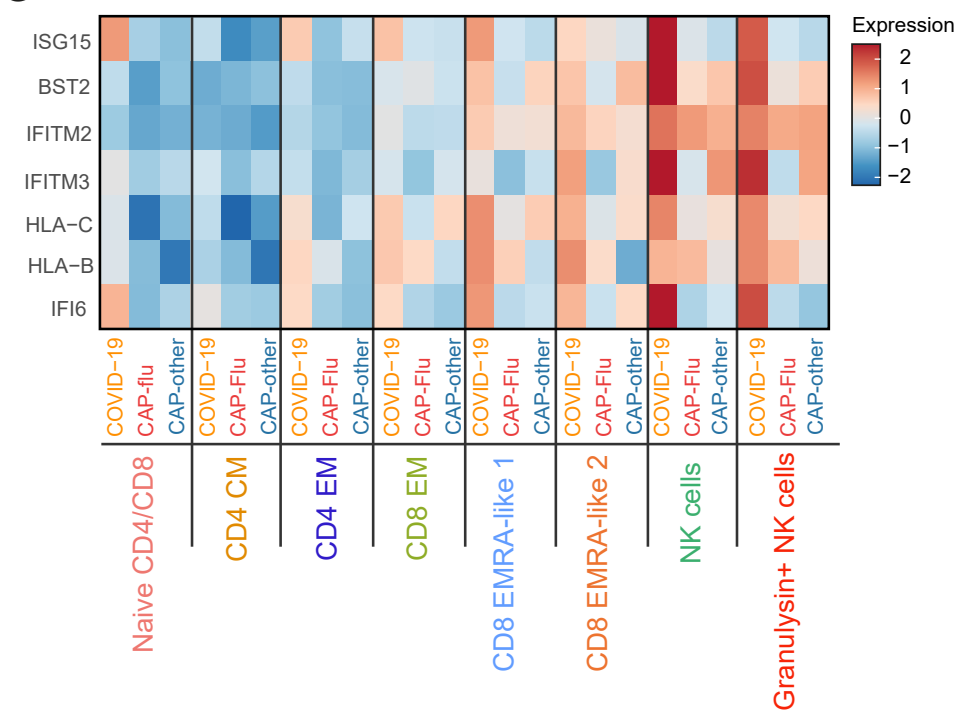

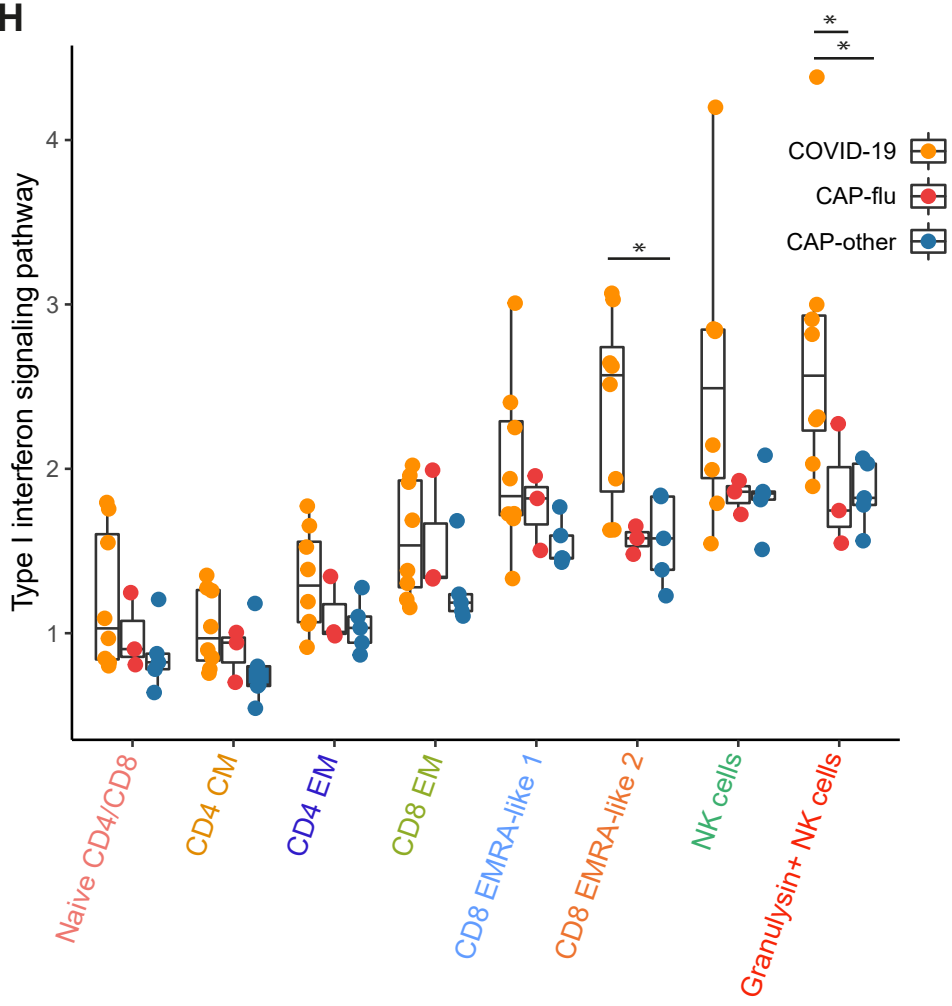


Fig. 4. CAP-flu is characterized by expansion of $T$ cells and NK cells expressing granulysin, while COVID-19 T and NK cells exhibit a clear type I interferon signature.

(a) UMAP depicting the T and NK cell clusters identified by the single-cell transcriptomic analysis of PBMCs from COVID-19, CAP-flu and CAP-other patients, where each dot represents a single cell with each color corresponding to a specific cell type cluster. The full UMAP of all identified clusters, the distribution per individual patient and the differentially expressed genes and proteins between all clusters are depicted in Supplementary Figs. 5 and 6. (b) Correlation plot depicting T and NK cell cluster enrichment in COVID-19, CAP-flu and CAP-other. Dot size proportional to Pearson's residual of the chi-squared test (i.e., reflecting the difference between the observed and expected proportion), while the color represents the degree of association from Pearson's chi-squared residuals (red means a positive association, blue means a negative association). See also Supplementary Fig. 5c for the correlation plot of all identified clusters. (c) Violin plots showing the expression of the top DEGs derived from comparing the two identified EMRA-like T cell clusters (adjusted $\mathrm{p}<0.05$ ). The expression of these genes in other $\mathrm{T}$ cell clusters are also depicted. See also Supplementary Fig. 6 for the top DEGs and surface protein expression differences between all identified cell clusters. (d) Violin plots showing the expression of the top DEGs between the two identified NK cell clusters (adjusted $\mathrm{p}<0.05$ ). (e) Graph depicting the DEGs identified when comparing COVID-19 cells and CAP-flu cells in the granulysin+ NK cell cluster. The X-axis depicts the average log fold change and the $\mathrm{Y}$-axis depicts the percent point difference between the proportion of cells expressing the gene in the COVID-19 group minus the proportion of cells expressing the gene in the CAP-flu group. All depicted DEGs are statistically significant after adjusting for multiple testing (Benjamini-Hochberg). (f) Bar plot showing Gene Ontology pathway analysis of the genes upregulated in granulysin+ NK cells from patients with COVID-19. X-axis shows the Benjamini-Hochberg adjusted - $\log 10$ p-value from the enrichment score analysis. (g) Heatmap 
showing the expression of genes in the type I interferon signaling pathway in all T and NK cell subsets, split between COVID-19, CAP-flu and CAP-other patients. (h) Box and whisker plots depicting the upregulation of the type I interferon signaling pathway in all $\mathrm{T}$ and NK cell subsets, split between COVID-19, CAP-flu and CAP-other patients. Statistical significance was determined using the two-sided Kruskal-Wallis test with post-hoc pairwise Dunn's test: * BHadjusted $\mathrm{p}<0.05$. 
A

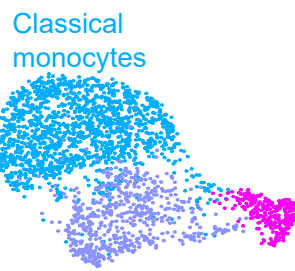

Non-classical monocytes

Intermediate monocytes
B

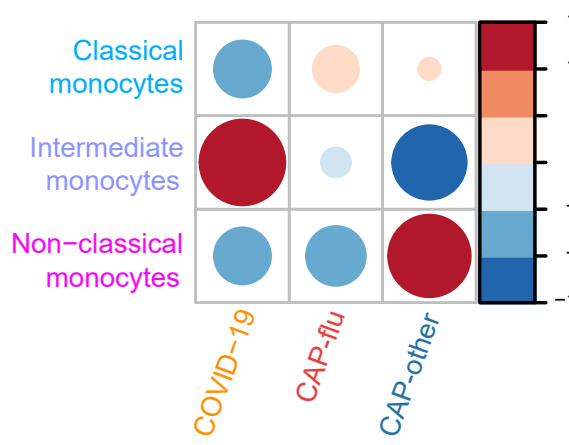

Classical monocytes

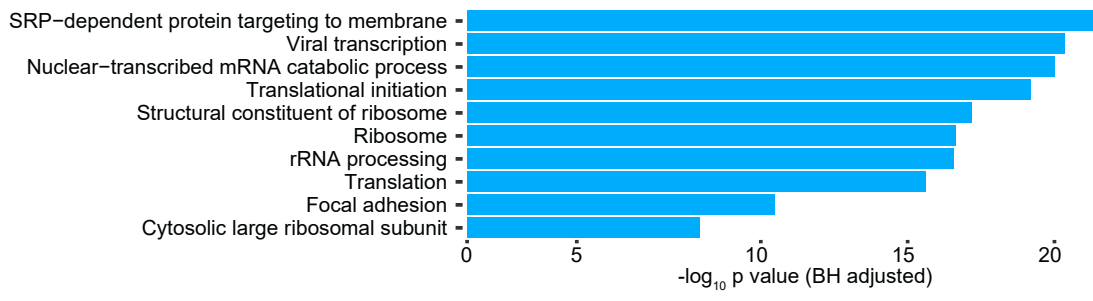

$E$

Intermediate monocytes

Type I interferon signaling pathway

Negative regulation of viral genome replication -

Response to interferon-beta -

Defense response to virus

Antigen presentation via MHC class I, TAP-dependent -

MHC class I protein complex
Response to virus

Antigen presentation via MHC class I, TAP-independent

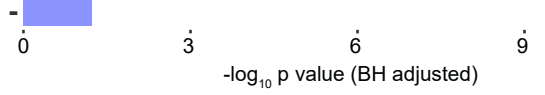

Non-classical monocytes

MHC class II protein complex . Immune response

Endoplasmic reticulum membrane

Antigen processing and presentation

ER to Golgi transport vesicle membrane

Interferon-gamma signaling pathway

MHC class II receptor activity -

Peptide antigen binding

Antigen processing via $\mathrm{MHC}$ class II

Transport vesicle membrane

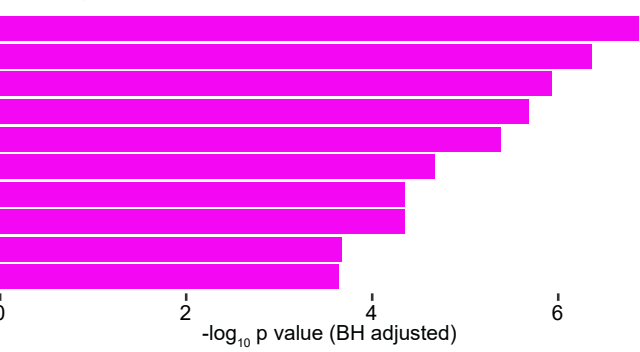

C

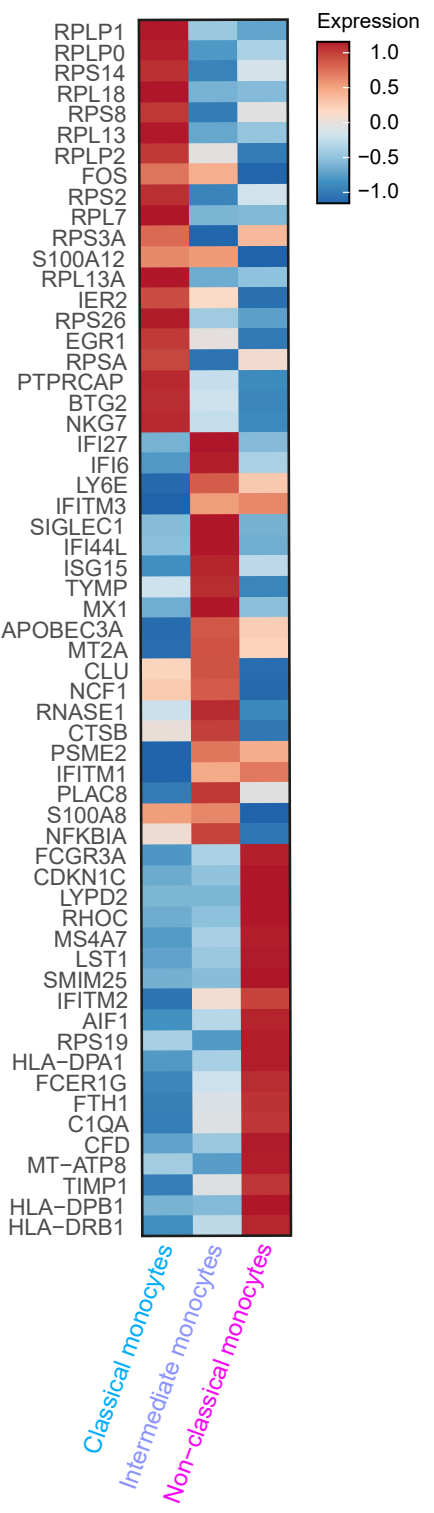


Fig. 5. Distinctive subset compositions and transcriptional profiles in monocytes from patients with COVID-19, CAP-flu and CAP-other. (a) UMAP depicting the monocyte cell clusters identified by the single-cell transcriptomic analysis of PBMCs from COVID-19, CAP-flu and CAP-other patients, where each dot represents a single cell with each color corresponding to a specific cell type cluster. The full UMAP of all identified clusters and the distribution per individual patient is depicted in Supplementary Figs. 5a,b. (b) Correlation plot depicting cluster enrichment in COVID-19, CAP and CAP-flu patients. Dot size proportional to Pearson's residual of the chi-squared test (i.e., reflecting the difference between the observed and expected proportion), while the color represents the degree of association from Pearson's chi-squared residuals (red means a positive association, blue means a negative association). (C) Heatmap showing the expression of the top DEGs (adjusted $\mathrm{p}<0.05$ ) between all three monocyte clusters. (d) Gene ontology pathway analysis of the upregulated DEGs in classical monocytes, (e) intermediate monocytes and (f) non-classical monocytes. Xaxis shows the Benjamini-Hochberg adjusted - $\log 10$ p-value from the enrichment score analysis. 


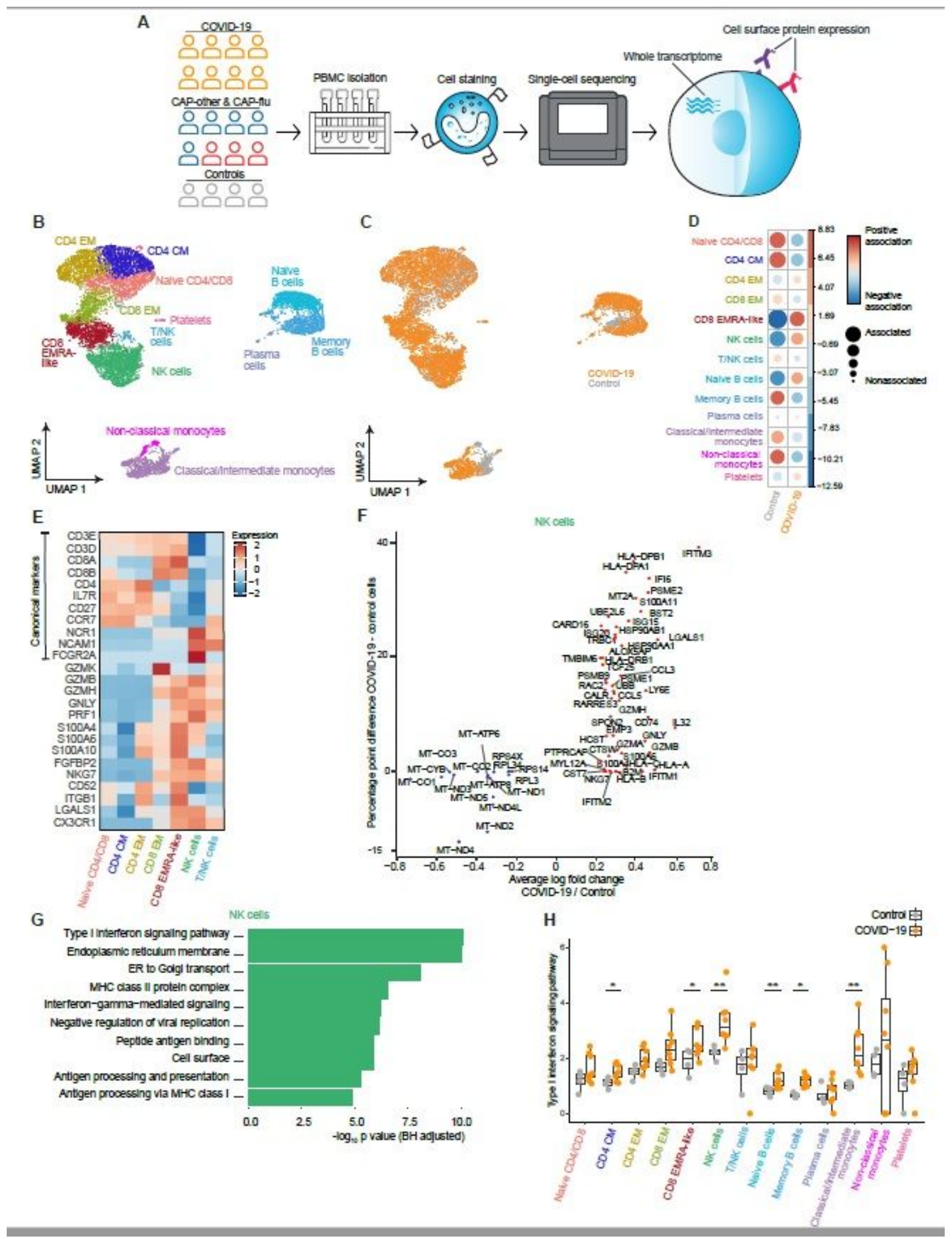

\section{Figure 1}

The immune response in COVID-19 is characterized by an expansion of CD8 EMRA-like T cells and type I interferon-stimulated NK cells, both demonstrating high cytotoxic potential. (a) Experimental overview: PBMCs from a matched cohort of hospitalized patients with CAP caused by SARS-CoV-2 (COVID-19), CAP 
caused by Influenza A or other pathogens, and non-infectious controls, were isolated and stained with a panel of oligonucleotide-tagged antibodies. Single-cell mRNA and surface protein expression were subsequently measured on a 10X Genomics platform. $(b, c)$ UMAPs depicting the clusters identified by the single-cell transcriptomic analysis of PBMCs from control subjects and patients with COVID-19, each dot representing a single cell. In the first UMAP (b) cells are colored by cell type cluster, whereas in the second UMAP (c) cells are colored by donor group. See also Supplementary Fig. 1. (d) Correlation plot depicting cluster enrichment in controls and COVID-19 patients. Dot size proportional to Pearson's residual of the chi-squared test (i.e., reflecting the difference between the observed and expected proportion), while the color represents the degree of association from Pearson's chi-squared residuals (red means a positive association, blue means a negative association). (e) Heatmap showing the expression of canonical genes and the top differentially expressed genes (DEGs) derived from comparing the CD8 EM and CD8 EMRA-like cell clusters (adjusted $p<0.05$ ). The heatmap also shows the expression of these genes in the other identified T and NK cell clusters. See also Supplementary Fig. 2. (f) Graph depicting the DEGs identified when comparing cells from COVID-19 patients and controls within the NK cell cluster. The Xaxis depicts the average log fold change and the $\mathrm{Y}$-axis depicts the percent point difference between the proportion of cells expressing the gene in the COVID-19 group minus the proportion of cells expressing the gene in the control group. All depicted DEGs are statistically significant after adjusting for multiple testing (Benjamini- Hochberg). (g) Bar plot showing Gene Ontology pathway analysis of genes upregulated in NK cells from patients with COVID-19 (relative to controls) identified in the analysis of panel G. X-axis shows the Benjamini-Hochberg adjusted -log10 p-value from the enrichment score analysis. (h) Box and whisker plots showing the enrichment of the type I interferon pathway in all cell subsets, split between COVID-19 patients and controls. The Y-axis depicts the enrichment score. Statistical significance was determined using the two-sided Wilcoxon ranksum test: ${ }^{*} p<0.05,{ }^{* \star} p<0.01$. 


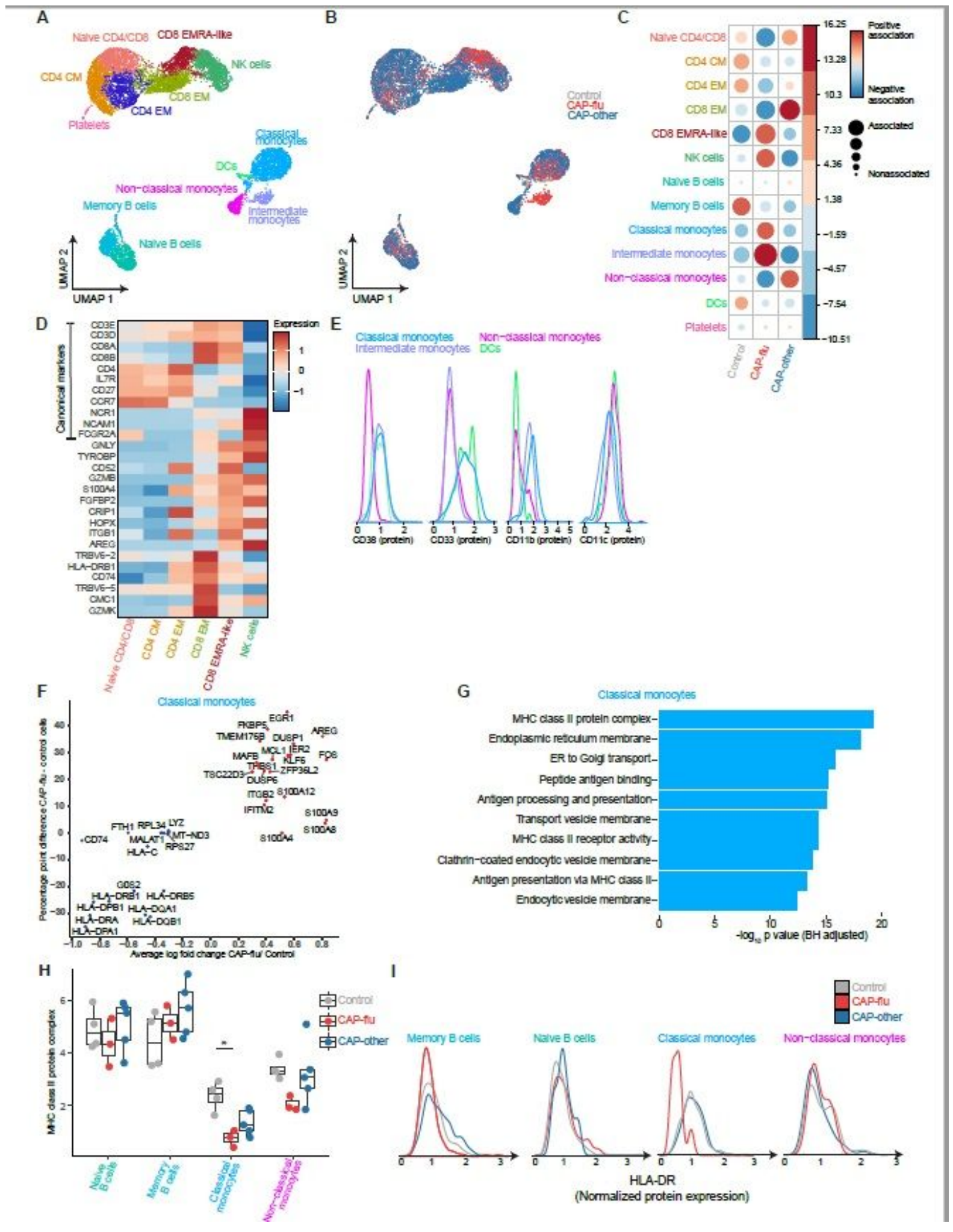

\section{Figure 2}

The peripheral immune features of T cells, NK cells and monocytes vary between CAP-flu and CAP-other. $(a, b)$ UMAPs depicting the clusters identified by the single-cell transcriptomic analysis of PBMCs from controls, CAP-flu and CAP-other patients, where each dot represents a single cell. In the first UMAP (a) cells are colored by cell type cluster, whereas in the second UMAP (b) cells are colored by donor group. See also Supplementary Fig. 3. (c) Correlation plot depicting cluster enrichment in controls, CAP-flu and 
CAP-other patients. Dot size proportional to Pearson's residual of the chi-squared test (i.e., reflecting the difference between the observed and expected proportion), while the color represents the degree of association from Pearson's chi-squared residuals (red means a positive association, blue means a negative association). (c) Heatmap showing the expression of canonical genes and the top differentially expressed genes (DEGs) derived from comparing the CD8 EM and CD8 EMRAlike cell clusters (adjusted $p$ $<0.05)$. The heatmap also shows the expression of these genes in the other identified $T$ and NK cell clusters. See also Supplementary Fig. 4. (e) Density plots showing the surface protein expression of CD38, CD33, CD11b and CD11c per myeloid cell cluster. (f) Graph depicting the DEGs in the classical monocyte cluster when comparing CAPflu patients versus controls. The $\mathrm{X}$-axis depicts the average log fold change and the $Y$-axis depicts the percent point difference between the proportion of cells expressing the gene in the CAP-flu group minus the proportion of cells expressing the gene in the control group. All depicted DEGs are statistically significant after adjusting for multiple testing (Benjamini- Hochberg). (g) Bar plot showing Gene Ontology pathway analysis of downregulated genes identified in the analysis of panel G. X-axis shows the Benjamini-Hochberg adjusted -log10 pvalue from the enrichment score analysis. (h) Boxplots depicting the downregulation of the MHC class II protein complex transcriptional pathway in naive B cells, memory B cells, classical monocytes and non-classical monocytes clusters, split between controls, CAP-flu and CAP-other patients. Statistical significance was determined using the twosided Kruskal-Wallis 37 test with post-hoc pairwise Dunn's test: * BH-adjusted $p<0.05$. (i) Density plot showing the normalized surface protein expression of HLA-DR on cells in naive B cells, memory B cells, classical monocytes and non-classical monocytes clusters, split between controls, CAP-flu and CAP-other patients. 
A
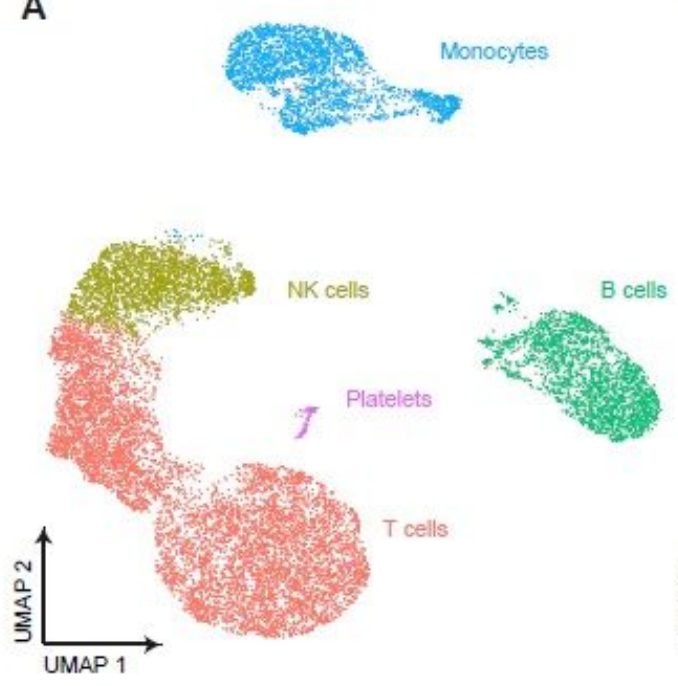

D

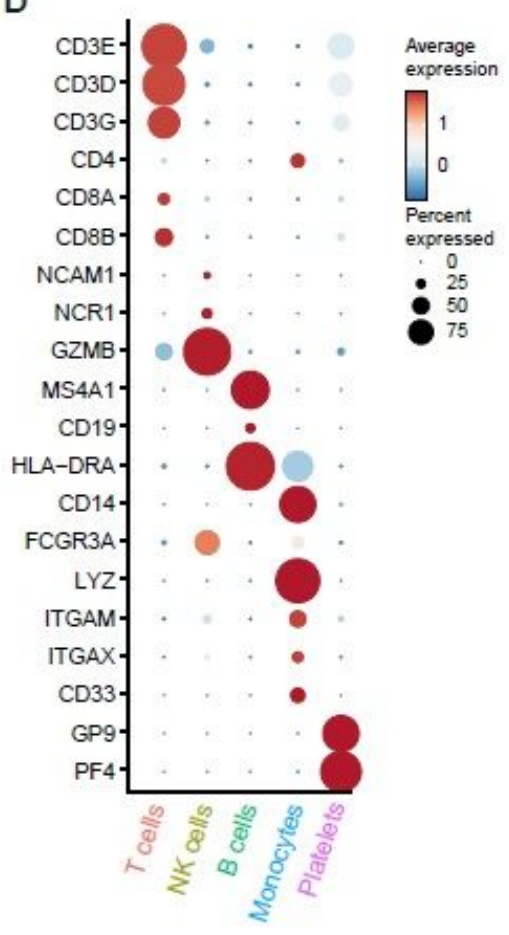

B

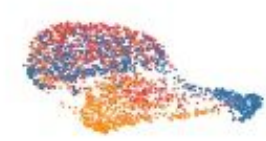

COVID-19
CAP-flu

CAP-other
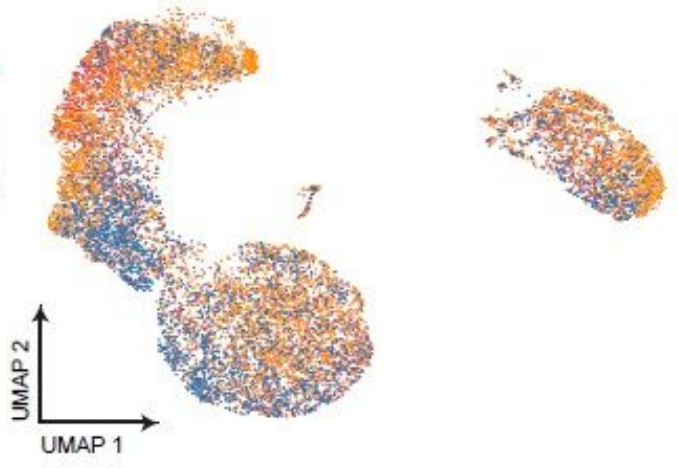

E

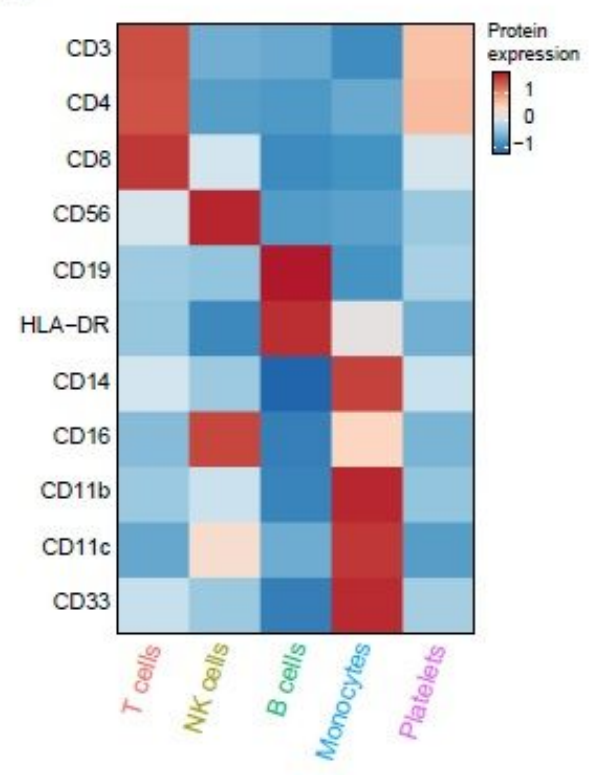

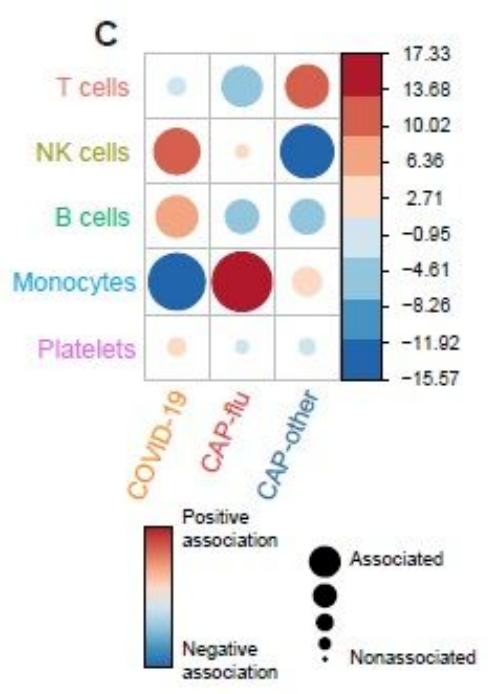

F

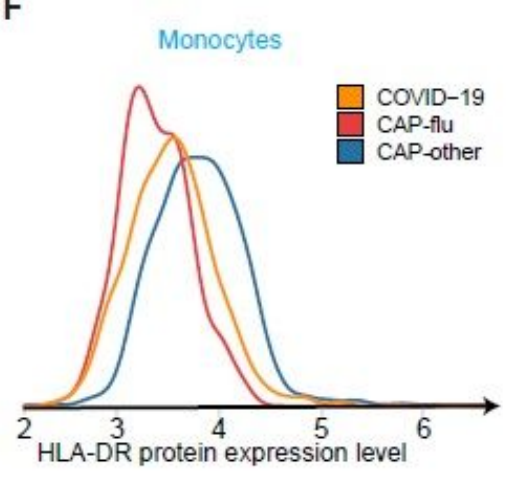

\section{Figure 3}

Divergent composition of major immune cell types in patients with COVID-19, CAP-flu and CAP-other. (a,b) UMAPs depicting the metaclusters identified by the single-cell transcriptomic analysis of PBMCs from controls, COVID-19, CAP-flu and CAP-other patients, where each dot represents a single cell. In the first UMAP (a) cells are colored by cell type cluster, whereas in the second UMAP (b) cells are colored by donor group. (c) Correlation plot depicting metacluster enrichment in COVID-19, CAP-flu or CAP-other patients. Dot size proportional to Pearson's residual of the chi-squared test (i.e., reflecting the difference between the observed and expected proportion), while the color represents the degree of association from Pearson's chi-squared residuals (red means a positive association, blue means a negative association). (d) Dot plot showing canonical genes per identified metacluster. Color indicates the normalized level of 
expression, while the dot size is proportional to the percentage of cells per cluster expressing the canonical gene. (e) Heatmap showing the expression of lineagedefining protein surface markers per metacluster. (f) Density plot showing the normalized surface protein expression of HLA-DR on the monocyte lineage, split between COVID-19, CAP-flu and CAP-other patients.

A
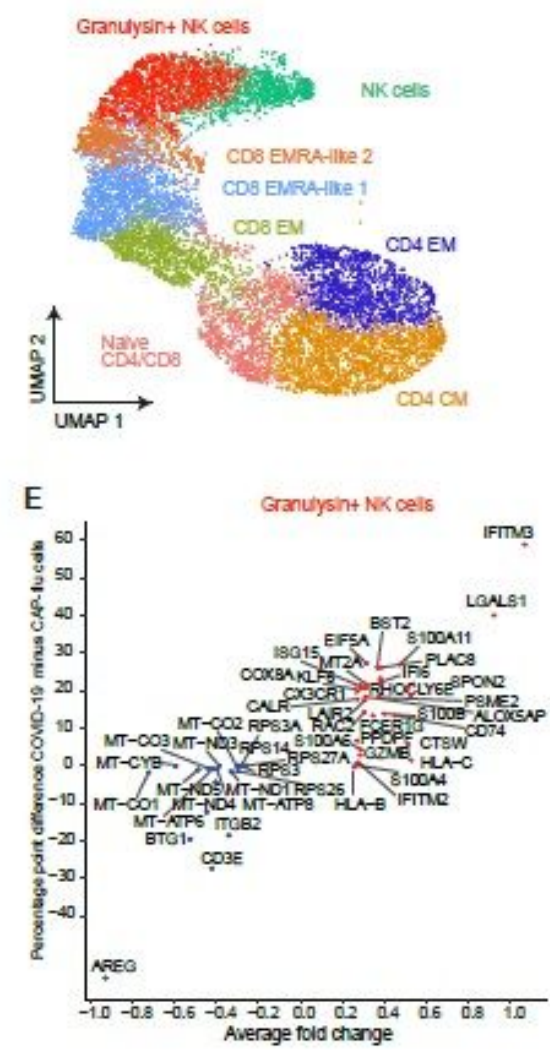

G

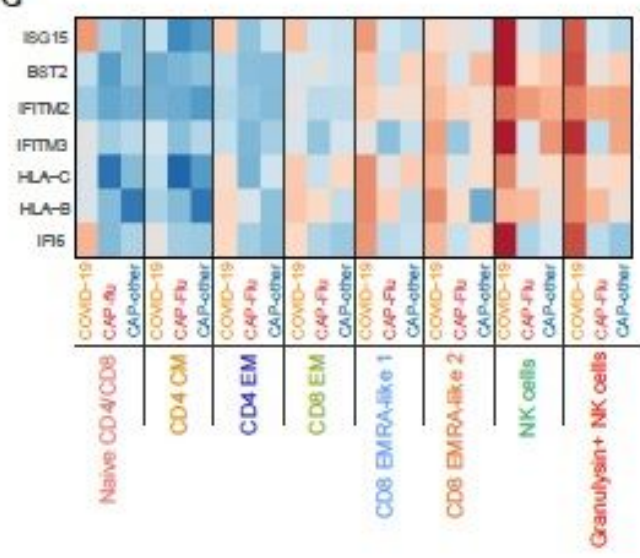

B

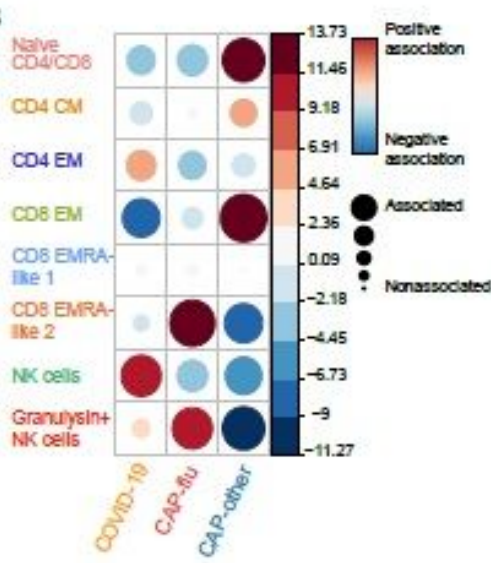

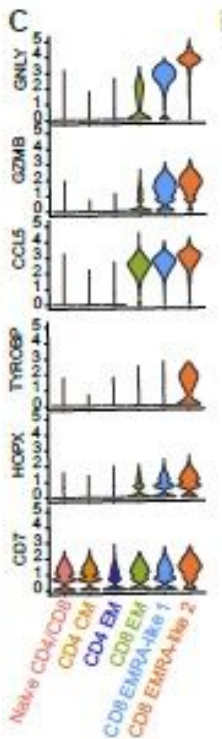

D

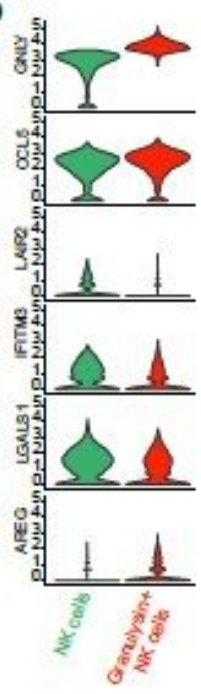

$\mathbf{F}$
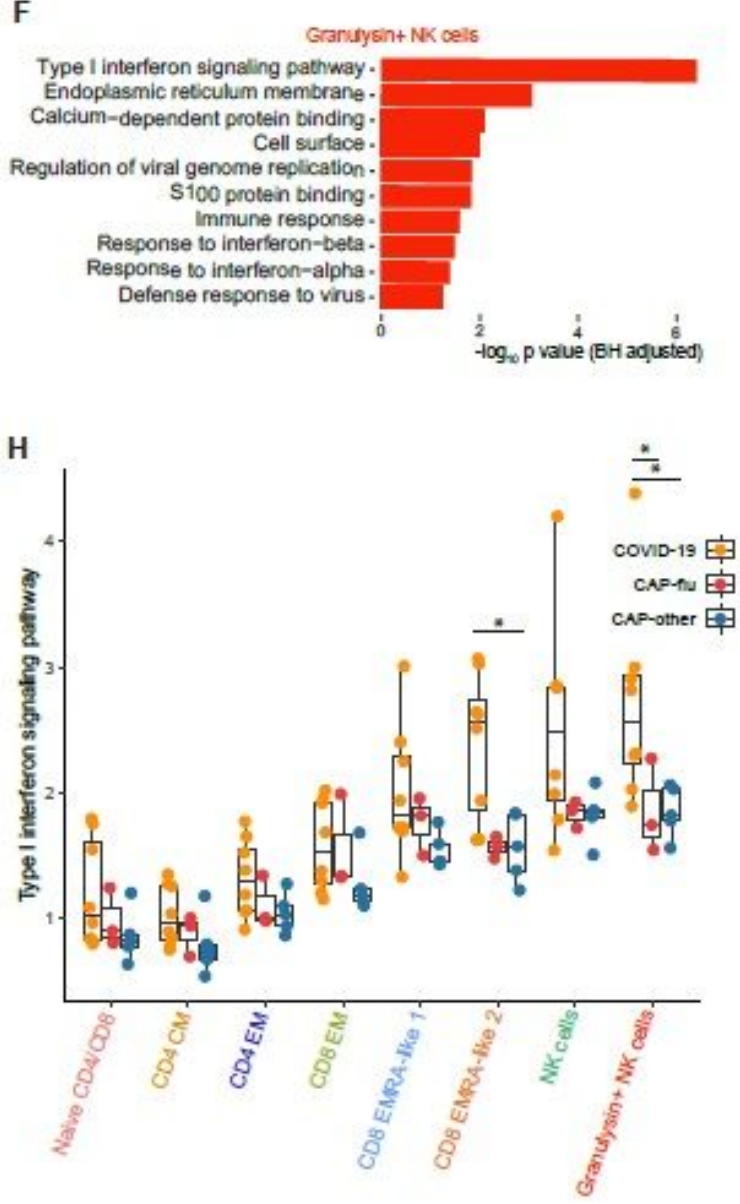

Figure 4 
CAP-flu is characterized by expansion of T cells and NK cells expressing granulysin, while COVID-19 T and NK cells exhibit a clear type I interferon signature. (a) UMAP depicting the T and NK cell clusters identified by the single-cell transcriptomic analysis of PBMCs from COVID-19, CAP-flu and CAP-other patients, where each dot represents a single cell with each color corresponding to a specific cell type cluster. The full UMAP of all identified clusters, the distribution per individual patient and the differentially expressed genes and proteins between all clusters are depicted in Supplementary Figs. 5 and 6. (b) Correlation plot depicting $T$ and NK cell cluster enrichment in COVID-19, CAP-flu and CAP-other. Dot size proportional to Pearson's residual of the chi-squared test (i.e., reflecting the difference between the observed and expected proportion), while the color represents the degree of association from Pearson's chi-squared residuals (red means a positive association, blue means a negative association). See also Supplementary Fig. 5c for the correlation plot of all identified clusters. (c) Violin plots showing the expression of the top DEGs derived from comparing the two identified EMRA-like T cell clusters (adjusted $p<0.05)$. The expression of these genes in other T cell clusters are also depicted. See also Supplementary Fig. 6 for the top DEGs and surface protein expression differences between all identified cell clusters. (d) Violin plots showing the expression of the top DEGs between the two identified NK cell clusters (adjusted $p<0.05$ ). (e) Graph depicting the DEGs identified when comparing COVID-19 cells and CAP-flu cells in the granulysin+ NK cell cluster. The X-axis depicts the average log fold change and the $Y$ axis depicts the percent point difference between the proportion of cells expressing the gene in the COVID-19 group minus the proportion of cells expressing the gene in the CAP-flu group. All depicted DEGs are statistically significant after adjusting for multiple testing (Benjamini-Hochberg). (f) Bar plot showing Gene Ontology pathway analysis of the genes upregulated in granulysin+ NK cells from patients with COVID-19. X-axis shows the Benjamini-Hochberg adjusted -log10 p-value from the enrichment score analysis. (g) Heatmap showing the expression of genes in the type I interferon signaling pathway in all $\mathrm{T}$ and NK cell subsets, split between COVID-19, CAP-flu and CAP-other patients. (h) Box and whisker plots depicting the upregulation of the type I interferon signaling pathway in all T and NK cell subsets, split between COVID-19, CAP-flu and CAP-other patients. Statistical significance was determined using the two-sided Kruskal-Wallis test with post-hoc pairwise Dunn's test: * BHadjusted $p<0.05$. 
A

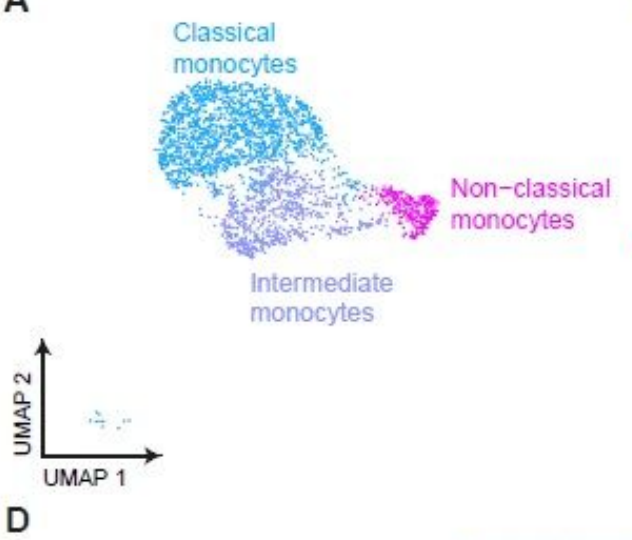

B

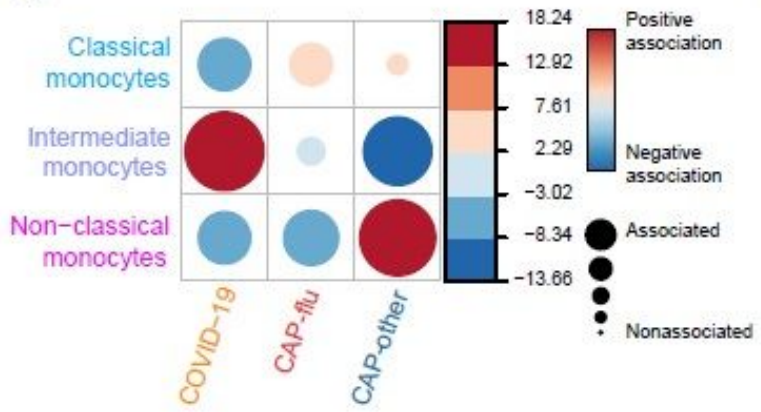

C

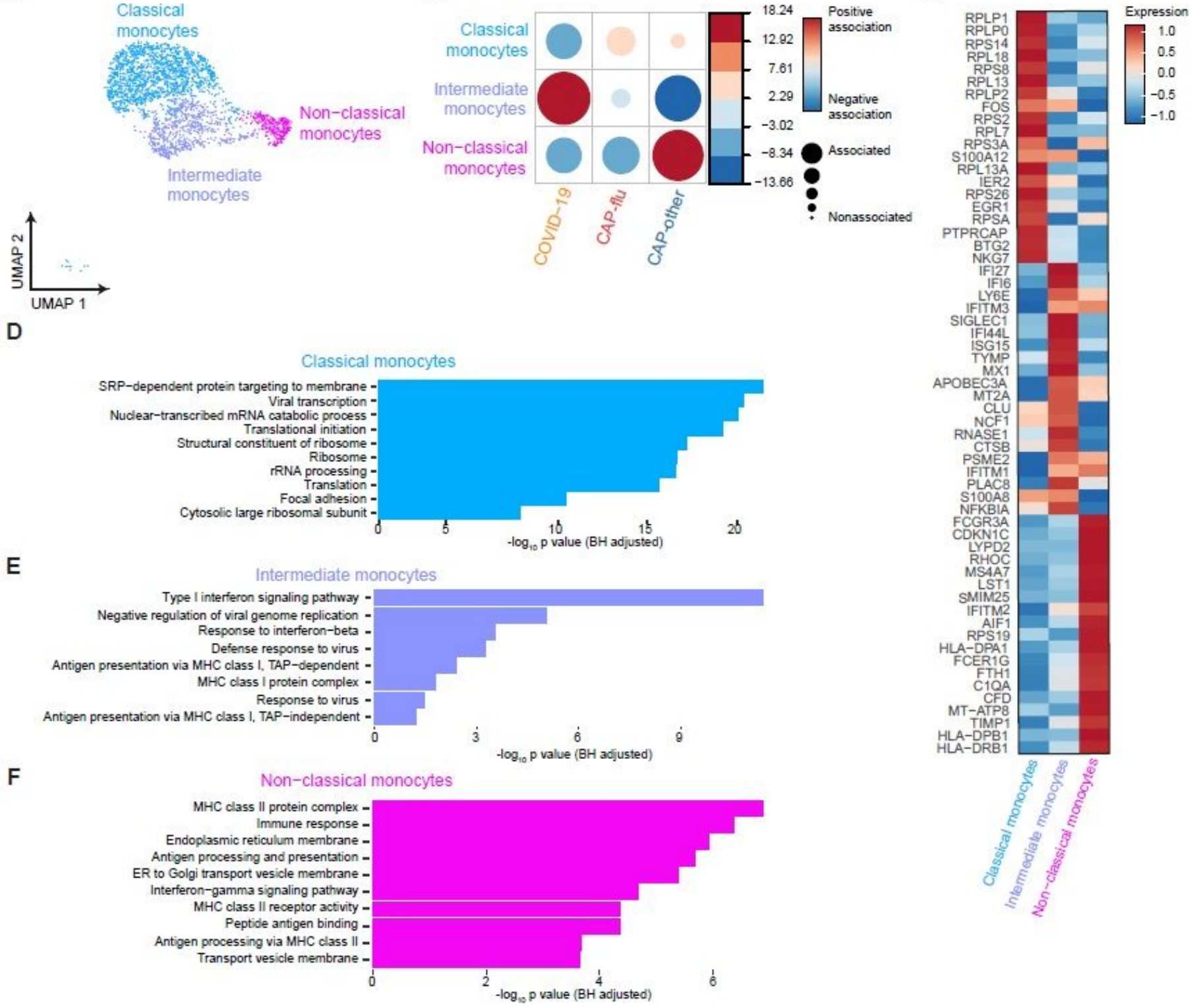

E

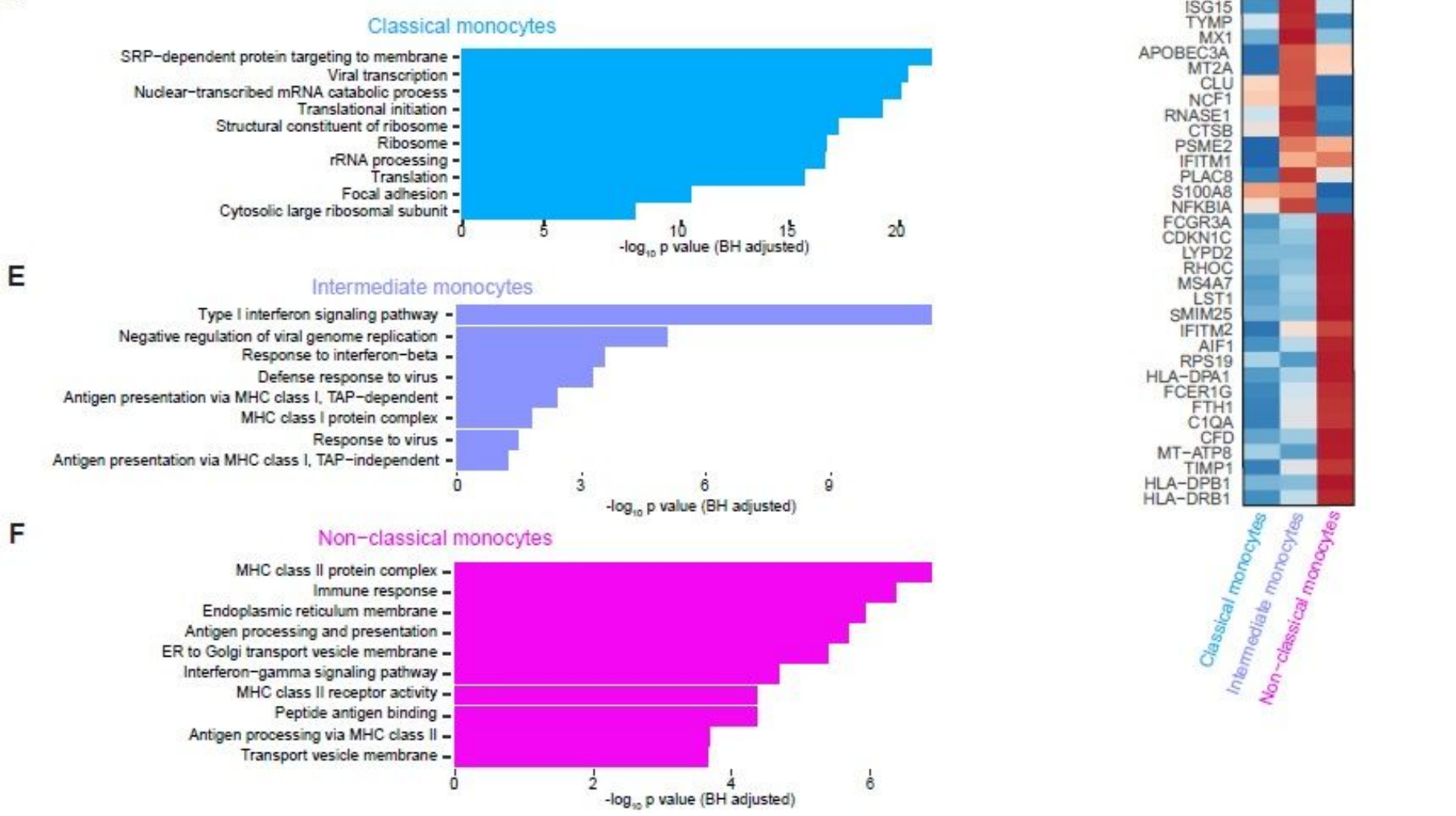

Intermediate monocytes

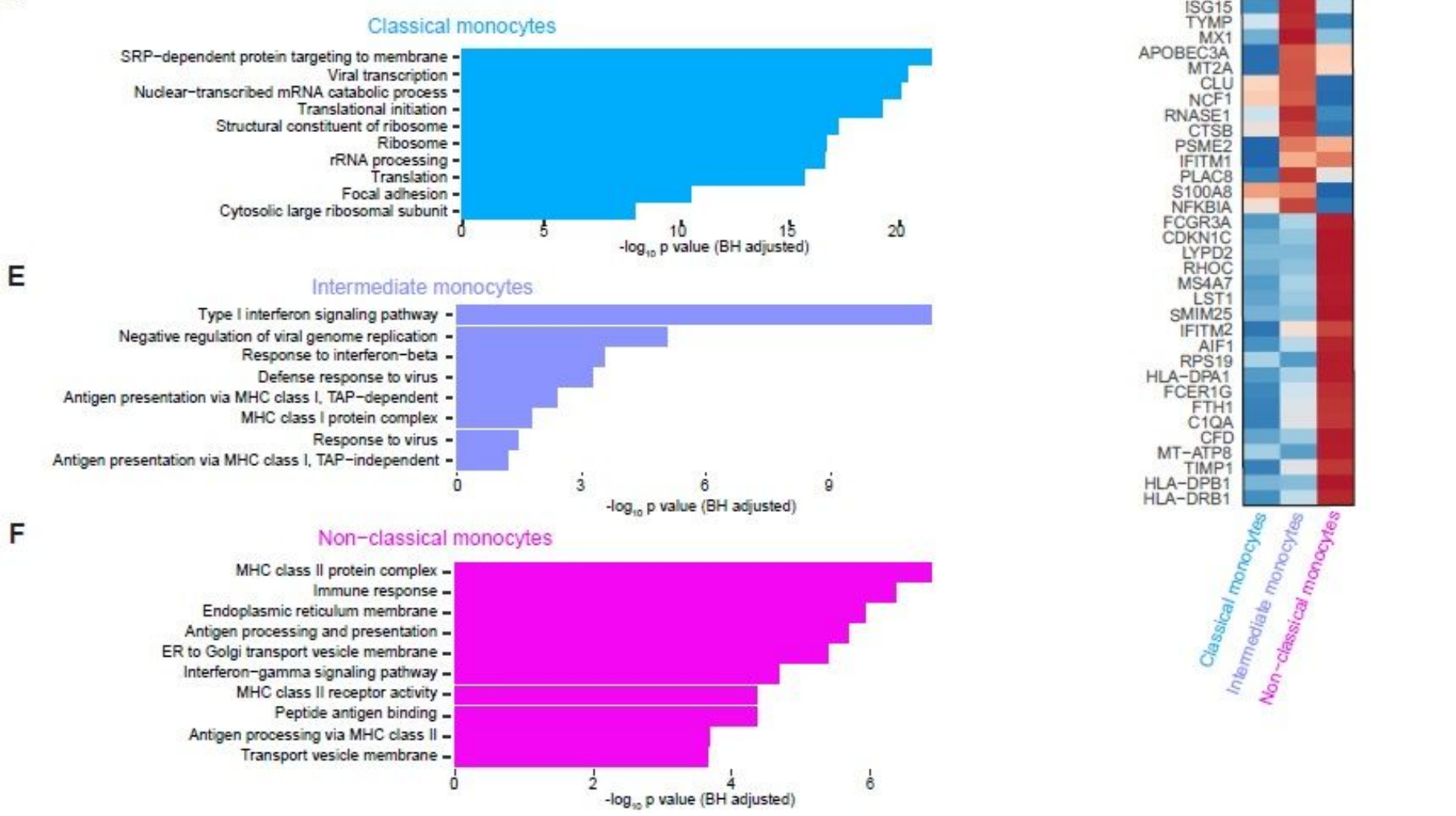

\section{Figure 5}

Distinctive subset compositions and transcriptional profiles in monocytes from patients with COVID-19, CAP-flu and CAP-other. (a) UMAP depicting the monocyte cell clusters identified by the single-cell transcriptomic analysis of PBMCs from COVID-19, CAP-flu and CAP-other patients, where each dot represents a single cell with each color corresponding to a specific cell type cluster. The full UMAP of all identified clusters and the distribution per individual patient is depicted in Supplementary Figs. 5a,b. (b) Correlation plot depicting cluster enrichment in COVID-19, CAP and CAP-flu patients. Dot size proportional to Pearson's residual of the chi-squared test (i.e., reflecting the difference between the observed and expected proportion), while the color represents the degree of association from Pearson's chi-squared residuals (red means a positive association, blue means a negative association). (C) Heatmap showing the expression of the top DEGs (adjusted $p<0.05$ ) between all three monocyte clusters. (d) Gene ontology pathway analysis of the upregulated DEGs in classical monocytes, (e) intermediate monocytes 
and (f) non-classical monocytes. Xaxis shows the Benjamini-Hochberg adjusted -log10 p-value from the enrichment score analysis.

\section{Supplementary Files}

This is a list of supplementary files associated with this preprint. Click to download.

- Supplementallnformationcombined.pdf

- SupplementalTableS1.xlsx 